\title{
Optimal transport in full-waveform inversion: Analysis and practice of the multidimensional Kantorovich-Rubinstein norm
}

\author{
Jérémie Messud $^{1}$, Raphaël Poncet ${ }^{2}$, Gilles Lambaré $^{1}$ \\ ${ }^{1}$ CGG, 27 avenue Carnot, 91341 Massy (France) \\ ${ }^{2}$ Formerly CGG \\ January 8, 2021
}

\begin{abstract}
In the last ten years, full-waveform inversion (FWI) has emerged as a robust and efficient high-resolution subsurface model-building tool for seismic imaging, with the unique ability to invert for complex models. FWI is based on the minimization of a cost function between observed and modelled data, the data space consisting in collections of time-series. Originally considering a least-squares cost function, the method suffered from high sensitivity to local minimums and was therefore of poor efficiency in handling large time shifts between observed and modelled data events. To tackle this problem, a common practice is to start the inversion using the low temporal frequencies of the data and selecting specific data events called diving waves. Complementary to this, the use of other cost functions has been investigated. Among these, cost functions based on optimal transport appeared appealing to possibly handle large time shifts between observed and modelled data events. Several strategies inspired by optimal transport have been proposed, taking into account the specificities of seismic data. Among them, the approach based on the Kantorovich-Rubinstein norm offers the possibility of the direct use of seismic data and an efficient numerical implementation allowing for a multidimensional (data coordinate space) application.

We present here an analysis of the Kantorovich-Rubinstein norm, discussing its theoretical and practical aspects. A key component of our analysis is the adjoint-source or data-space gradient of the cost function (converted into the model-space gradient within FWI). We highlight its piecewise linearity, analyze its frequency content and amplitude, and emphasize the benefit of having a multidimensional implementation. We give practical rules for setting the tuning parameters. Our set of synthetic and field data examples demonstrate the improvements brought by the use of the Kantorovich-Rubinstein norm over least-squares FWI, and highlight the improvements brought by the multidimensional approach over the one-dimensional one.
\end{abstract}

Keywords: Optimal transport, multimensional Kantorovich-Rubinstein norm, full-waveform inversion, seismic imaging

Submitted to: Inverse Problems

\section{Introduction}

Full-waveform inversion (FWI) was proposed in the early 80's as a data fitting process aimed at inverting for a subsurface model (Lailly, 1983; Tarantola, 1984). It is based on a cost function between observed and modelled data, initially the least-squares (LSQ) one, minimized through a non-linear iterative local optimization scheme. The observed data consists in collections of timeseries or "traces" (generated by a source and measured at the Earth's surface after propagation in the subsurface), called common shot gathers. The modelled data is computed by solving the wave-equation in a given subsurface model (Virieux and Operto, 2009).

After initial successes, FWI encountered difficulties related to its computational cost and the building of the long spatial wavelength components of the subsurface model from seismic data events called reflected waves (Tarantola, 2005). The investigations continued, moving from the inversion of reflected waves to the inversion of other seismic data events called transmitted and diving waves, and starting the inversion using the low temporal frequencies of the data and frequently an 
offset (or traces) selection (Pratt, 1990, 1999; Bunks et al., 1995; Sirgue and Pratt, 2004). From the mid-2000's the successes and potential of these approaches gave industrial perspectives for complex subsurface imaging (Operto et al., 2004; Brenders and Pratt, 2007; Virieux and Operto, 2009). The computational cost remained a blocking factor in the case of 3D subsurface models, but this was rapidly overcome thanks to specific implementations and the increase in computational capabilities (Vigh and Starr, 2007; Sirgue et al., 2009; Plessix, 2009). Since the late-2000's, FWI has been established as an essential component of the subsurface (or "velocity") model building toolbox used in the seismic imaging industry. Its capability to recover complex and high-resolution subsurface models has made it a favored tool.

However, issues still remained. Firstly, the LSQ cost function is very sensitive to local minimums because of the oscillatory nature of seismic data, so that the local optimization can easily get trapped into a local minimum (Virieux and Operto, 2009). This is called the cycle-skipping problem. Secondly, the LSQ cost function is very sensitive to amplitudes so that spurious inversion results can be obtained when the assumptions used in the modelling do not make it possible to "quantitatively reproduce" the amplitudes in the data (most implementations of FWI are currently based for example on an acoustic modelling while the subsurface is elastic). As mentioned above, starting the optimization from a good initial model, inverting first the low temporal frequency components of the data (Bunks et al., 1995) and using mainly diving waves reduces the sensitivity to cycle-skipping (Tarantola, 2005), while data preprocessing (in particular trace normalization) reduces the sensitivity to amplitude. However, these ad-hoc strategies do not completely solve the problem and require a careful implementation. As a consequence, a search for complementary solutions, in particular in terms of more suitable cost functions, has emerged as a very active research field. The expected features of alternative cost functions are: an increased sensitivity to the kinematic information contained in the data (or time shifts between observed and modelled data events) and a relaxed sensitivity to the amplitude information. Many proposals were made around the early-2010's (Shin and Ha, 2008; Van Leeuwen and Mulder, 2010; Luo and Sava, 2011; Warner and Guasch, 2016), following some pioneering work (Luo and Schuster, 1991). The first proposal of a cost function inspired from optimal transport (OT) was made by Engquist and Froese (2014).

Métivier et al. (2016b) provide the state-of-the-art of OT-based cost functions and their first usage in FWI. They mention the original work of Gaspard Monge (18th century) aiming at finding the optimal way to transport piles of sand by minimizing the expended energy or cost (Monge, 1781). Mathematically, the distribution of masses of initial and final piles of sand are described by probability density functions (PDFs) (positive, with unit "mass"). The transportation is described as a mapping between the initial and final PDFs. Minimizing the transportation cost involves the Monge-Ampère equation in the general case (Villani, 2008). Various reformulations of the original problem exist, that can usually be related to Wasserstein distances. Regarding FWI, the approach proposed by Engquist and Froese (2014); Engquist et al. (2016); Yang and Engquist (2018); Yang et al. (2018) and recently applied to field data by Wang and Wang (2019) is based on a 2-Wasserstein distance. Because of the computational cost of the resolution of the Monge-Ampère problem, the approach currently only affords for a trace-by-trace comparison in the 2-Wasserstein distance for large scale applications, not a comparison of a set of traces as in a common shot gather. So, the formulation does not use the property of OT-based cost functions to eventually be multidimensional in the data coordinate space (not to be confused with the dimensionality of the subsurface model), i.e. to account for correlations between traces (multidimensional) and not only within one trace (one-dimensional). Also, within this formulation, the requisite for positive values and mass conservation imposes ad-hoc transformations of seismic traces.

In this context, Métivier et al. (2016a, 2016b, 2016c) proposed an alternative to this first family of OT-based cost functions, allowing the ad-hoc transformations of seismic traces to be bypassed. They started from the dual formulation of the 1-Wasserstein distance, called the KantorovichRubinstein (KR) formulation, that leads to a maximization problem over 1-Lipschitz functions. More specifically, they proposed to compute a distance between seismic data using the so-called KR norm (Hanin, 1992; Villani, 2003; Lellmann et al., 2014). Adding a bounding constraint allows the direct use of the seismic data without any transformation. An important feature is that a version of the KR norm exists that can be very efficiently computed using the simultaneous descent method of multipliers (SDMM) iterative algorithm (Combettes and Pesquet, 2011; Métivier et al., 2016b). As 
a consequence, the use of the KR norm is in practice not limited to a trace-per-trace comparison. A multidimensional formulation in the data coordinate space is feasible for large scale applications, which can account for correlations in the offset direction between traces in a common shot gather, which is not the case with an approach based on a 2-Wasserstein distance as Engquist and Froese (2014). As shown in Métivier et al. (2016a) the KR norm and its numerical approximation does not lead to a full convexity when comparing two shifted Ricker wavelets. It however becomes more convex when the number of iterations of the SDMM algorithm increases (the secondary minima tend to be pushed out) and outperforms the convexity of LSQ while keeping a sharp valley of attraction (it can tend at convergence to a cost function valley almost twice wider than the LSQ one). Recently, this approach led to numerous successful industrial FWI applications, see e.g. Poncet et al. (2018); Messud and Sedova (2019); Sedova et al. (2019); Hermant et al. (2019); Carotti et al. (2020); Hermant et al. (2020). Compared to LSQ FWI, an interesting reduction in sensitivity to cycle-skipping has been observed, together with an improved structural consistency in inverted subsurface models. These behaviors can be related to the specific nature of the corresponding "adjoint-source" or data-space gradient of the cost function (converted within FWI into the subsurface model-space gradient through back-propagation (Virieux and Operto, 2009)). Compared to the LSQ FWI adjoint-source, the KR FWI adjoint-source exhibits an enhancement in low frequencies, balancing of amplitudes and an increase in coherency along the events (i.e. along a direction called "moveout") when using the multidimensional formulation (Messud and Sedova, 2019).

This paper deals with the use of the KR norm in FWI, in the continuation of the work of Métivier et al. (2016a, 2016b, 2016c). We firstly focus the analysis on the KR adjoint-source. We clarify theoretical aspects related to the establishment of the expression of the adjoint-source. We rigorously define the "texture" of the KR adjoint-source (piecewise linearity, lower frequency content, reduced amplitude dynamics) and emphasize the benefit of having a multidimensional implementation. We demonstrate the interest of the KR adjoint-source for FWI in terms of "physical" characteristics, explain the meaning of the tuning parameters (critical for a successful implementation) and give a set of practical rules for setting these parameters. Then, we present a set of synthetic and field data FWI examples demonstrating the effectiveness of the approach over LSQ FWI, as well as the benefit of the multidimensional KR FWI approach over the onedimensional one. Finally, to overcome some limitations, we discuss the possibility to combine the approach with a kinematic transformation applied to data, like the "graph-space" OT one proposed by Métivier et al. (2019).

Before presenting our contributions, we start with reminders on FWI and OT formalisms (§2).

\section{Theory}

\subsection{FWI and the challenge of cycle-skipping}

We denote by $\mathbb{R}^{(X)}$ the family of real functions $f: X \rightarrow \mathbb{R}$ indexed on a space $X$ (finite or infinite dimensional) that is measurable for a measure $\mu$. A seismic survey corresponds to a series of recorded shots, each shot producing a collection of traces called common shot gather. The data space related to one common shot gather is

$$
D(X) \subset \mathbb{R}^{(X)} .
$$

It is indexed on $X=\left[H_{x l}^{\min }, H_{x l}^{\max }\right] \times\left[H_{i n l}^{\min }, H_{i n l}^{\max }\right] \times[0, T] \subset \mathbb{R}^{3}$ that represents the set related to the positions in a common shot data. $T$ denotes the maximum recording time of each trace. $H_{x l}^{\min }, H_{x l}^{\max }$ and $H_{i n l}^{\min }, H_{i n l}^{\max }$ denote the minimum and maximum receiver positions, respectively in the crossline and inline directions, that index the position of the traces within the common shot data. $x=\left[x_{x l}, x_{\text {inl }}, x_{t}\right]^{t} \in X$ represents a 3 dimensional (3D) vector, where ${ }^{t}$ denotes the transpose and $x_{x l}, x_{i n l}, x_{t}$ are scalars. In the following, we call $X$ the data "coordinate space", considered as continuous, with the associated Lebesgue measure denoted by $\mu(x)$. However, the considerations 
in this article generalize to discrete sets $X$ simply considering $\mu(x)$ to be the counting measure $\dagger$.

A seismic observed shot data, here considered as a scalar field measured at the Earth's surface during a seismic experiment, is described by $f_{\text {obs }}: X \rightarrow \mathbb{R}, f_{\text {obs }} \in D(X)$. In FWI, modelled shot data is produced by solving the wave-equation in a given subsurface model $m \in M$, where $M$ denotes the model space $\ddagger$, and extracting the result at the Earth's surface. Such a modelled shot data is described by $f[m]: X \rightarrow \mathbb{R}, f[m] \in D(X)$. The FWI problem consists in finding $m^{*} \in M$ which best explains all the observed common shot data, i.e. makes $f\left[m^{*}\right]$ as close as possible to $f_{o b s}$ for all shots from the point of view of a given similarity measure in the data space (Tarantola, 2005). We denote such a similarity measure, also called cost function, by a functional $J:[D(X) \times D(X)]^{N_{\text {shots }}} \rightarrow \mathbb{R}^{+}$, where $N_{\text {shot }}$ represents the number of shots; the latter dependency is implicit in the following to lighten the notations. We resolve

$$
m^{*}=\underset{m \in M}{\operatorname{argmin}} J\left(f[m], f_{o b s}\right) .
$$

The model space dimensionality requires to use an iterative local optimization scheme, based on the model-space gradient, i.e. $\partial J\left(f[m], f_{\text {obs }}\right) / \partial m=\int_{X} \partial f[m](x) / \partial m \times \partial J\left(f, f_{o b s}\right) /\left.\partial f(x)\right|_{f=f[m]} d \mu(x)$. Firstly, a forward propagation consists of resolving the wave-equation to compute the forwardpropagated wavefield, extracted at the Earth's surface to give $f[m]$. Secondly, the gradient in the data-space or "adjoint-source" is computed, i.e.

$$
\left.\frac{\partial J\left(f, f_{o b s}\right)}{\partial f(x)}\right|_{f=f[m]}
$$

that represents the sensitivity of the cost function to the data. Thirdly, the adjoint-source is "converted" into the model-space gradient $\partial J\left(f[m], f_{o b s}\right) / \partial m$. To that aim, as the dimensionality of the problem (in data and model spaces) makes a $\partial f[m](x) / \partial m$-based conversion unfeasible, FWI uses the adjoint-state method (Plessix, 2006; Virieux and Operto, 2009). The adjoint-source is back-propagated using the time-reversed wave-equation to obtain the back-propagated wavefield. Next, the forward-propagated wavefield and the second temporal derivative of the back-propagated wavefield are zero-lag cross-correlated in the time direction (when the wave-equation is considered in the time domain). This gives as a result the model-space gradient (Plessix, 2006). The latter may then be preconditioned and finally a line-search is applied to give the correct magnitude to the resulting descent direction. These are the main features of the FWI descent direction computation (Plessix, 2006; Virieux and Operto, 2009). The important point for this article is that the choice of the cost function $J$ affects the method directly only through the adjoint-source computation, eq. (3). So, to understand the interest of a given cost function, interpreting how the adjoint-source is impacted is a key.

Common FWI cost functions (and thus adjoint-source forms) are based on $L_{p}$ norms $(p \geq 1)$ through

$$
\begin{aligned}
& J\left(f[m], f_{o b s}\right)=\left\|f[m]-f_{o b s}\right\|_{p}^{p} \text { with }\|f\|_{p}=\left(\int_{X}|f(x)|^{p} d \mu(x)\right)^{1 / p} \\
& \Rightarrow \frac{\partial J\left(f, f_{o b s}\right)}{\partial f(x)}=\operatorname{sign}\left(f(x)-f_{o b s}(x)\right) \times\left|f(x)-f_{o b s}(x)\right|^{p-1},
\end{aligned}
$$

for $D(X) \subseteq L^{p}(X)$ where $L^{p}(X)$ denotes the space of functions whose moments of order $p$ are integrable. At the limit $p \rightarrow \infty$, the "uniform norm" is defined by $\|f\|_{\infty}=\max _{x \in X}|f(x)|$. The $p=2$ case leads to the most common FWI cost function, called least-squares (LSQ). The LSQ adjoint-source is equal to $f[m]-f_{\text {obs }}$ and called the "residual".

Let us come back to the (data) coordinate space $X$. As already mentioned, the dimensionality of $X$ is 3 for common shot data (time direction, and receiver crossline and inline directions). However, if an application utilizes the data trace-per-trace independently, the

$\dagger$ For instance taking $X=\left\{H_{x l}\left(i_{x l}\right), i_{x l}=1 . . N_{x l}\right\} \times\left\{H_{i n l}\left(i_{i n l}\right), i_{i n l}=1 . . N_{i n l}\right\} \times\left\{T\left(i_{t}\right), i_{t}=1 . . N_{t}\right\}$, with $H_{x l}(1)=H_{x l}^{m i n}, H_{x l}\left(N_{x l}\right)=H_{x l}^{\text {max }}, H_{i n l}(1)=H_{i n l}^{m i n}, H_{i n l}\left(N_{i n l}\right)=H_{i n l}^{\max }, T(0)=0$ and $T\left(N_{t}\right)=T . N_{x l} \times N_{i n l}$ then represents the total number of traces in the considered common shot data. $N_{x l} \times N_{i n l} \times N_{t}$ represents the total number of samples in the common shot data, i.e. the dimensionality of the data space $D(X)$, typically equal to $10^{6}-10^{9}$. The associated counting measure is defined by $\mu(x)=\sum_{i_{x l}=1}^{N_{x l}} \sum_{i_{i n l}=1}^{N_{i n l}} \sum_{x_{t}=1}^{N_{t}} \delta\left(x_{x l}-H_{x l}\left(i_{x l}\right)\right) \delta\left(x_{i n l}-\right.$ $\left.H_{\text {inl }}\left(i_{\text {inl }}\right)\right) \delta\left(x_{t}-T\left(i_{t}\right)\right)$.

$\ddagger$ Typically a grid with $10^{6}-10^{8}$ samples. 
effective dimensionality of $X$ becomes 1 (time direction only enters into the computation). If an application utilizes the data inline-per-inline independently, the effective dimensionality of $X$ becomes 2 (inline and time directions only enter into the computation). Hereafter, when we mention multidimensionality (multiD), we refer to the effective dimensionality of the (data) coordinate space $X$ (not to be confused with the dimensionality of the data space $D(X)$ or of the model space).

It is obvious from from eq. (4) that $L_{p}$-based cost functions measure a point-wise similarity so that their effective dimensionality in the coordinate space is 0 ; in other words, no correlation between different positions in the time-direction are explicitly accounted for in the cost function (of course, some correlations are implicitly accounted for by inverting first the low temporal frequency components of the data and progressively adding higher frequencies). This is a fundamental reason for the high sensitivity to cycle-skipping of the $L_{p}$-based cost functions. Another potential issue is that these cost functions tend to be very sensitive to amplitudes; this sensitivity can somewhat be reduced by making $p$ very close to 1 . Indeed, all amplitudes are equalized in the $L_{1}$ adjoint-source, $\operatorname{sign}\left(f[m]-f_{o b s}\right)$, eq. (4). But this can lead to convergence difficulties and is still plagued by cycle-skipping.

In contrast, OT-based cost functions are explicitly at least 1D in the coordinate space, allowing them to explicitly account for correlation at least in the time direction, i.e. between an observed full trace and the corresponding modelled full trace. This provides more sensitivity to time shifts between the data events, thus more robustness to cycle-skipping (Engquist and Froese, 2014; Yang et al., 2018; Métivier et al., 2016c; Poncet et al., 2018; Messud and Sedova, 2019). Furthermore, these cost functions can even be multiD (2D or 3D) in the coordinate space, allowing the exploitation of the correlations between many observed traces and the corresponding modelled traces, i.e. the coherency in the moveout direction; also, OT-based cost functions are less sensitive to amplitudes (Métivier et al., 2016c; Poncet et al., 2018; Messud and Sedova, 2019). They thus seem a very good candidate to overcome the limitations of $L_{p}$-based cost functions.

However, there are two possible obstacles to the application of OT to large scale FWI problems. OT is originally designed to compare probability distributions, not signed and oscillatory functions like the seismic data. Also, the OT algorithm originally proposed for FWI (Engquist and Froese, 2014) is for now viable in an industrial context only for a 1D coordinate space, i.e. trace-per-trace comparison. In the next section, we review the general OT formalism and a formulation recently proposed by Métivier et al. (2016b, 2016c) that can overcome these obstacles.

\subsection{From optimal transport to the Kantorovich-Rubinstein norm}

We plan to use OT within the FWI framework. The latter is deterministic whereas OT is originally a statistical formalism that measures a similarity between probability distributions (Ambrosio et al., 2005). So, let us start with some probability-related considerations. We consider probability distributions that are absolutely continuous with respect to the measure $\mu(x)$. These probability distributions can be represented by PDFs, denoted by $\rho: X \rightarrow \mathbb{R}^{+}$. They have a unit "mass": $\int_{X} \rho(x) d \mu(x)=1$.

We provide the coordinate space $X$ with a metric $d: X \times X \rightarrow \mathbb{R}^{+}$. We consider $d^{p}(x, y)$ to represent the cost of displacing information from $x$ to $y$ in the coordinate space. $\mathcal{P}^{p}(X)$ denotes the space of PDFs with finite $d$-moment of order $p$, i.e. $\int_{X} d^{p}\left(x^{\prime}, x\right) \rho(x) d \mu(x)<+\infty$ for some (thus any) $x^{\prime} \in X$ (Villani, 2008). OT allows the computation of a cost of "transporting" the $\mathrm{PDF} \rho_{1} \in \mathcal{P}^{p}(X)$ onto the PDF $\rho_{2} \in \mathcal{P}^{p}(X)$ based on the coordinate space cost $d^{p}$. Kantorovich's formulation represents the most general OT form (Kantorovich, 1942; Villani, 2008; Ambrosio and Gigli, 2009):

$$
W_{d^{p}}^{p}\left(\rho_{1}, \rho_{2}\right)=\min _{\pi \in \Pi\left(\rho_{1}, \rho_{2}\right)} \int_{X \times X} d^{p}(x, y) \pi(x, y) d \mu(x) d \mu(y)
$$

where $\pi$ represent "transference plans" belonging to the set of PDFs on $X \times X$ that have marginals $\rho_{1}$ and $\rho_{2}$ :

$$
\begin{aligned}
\Pi\left(\rho_{1}, \rho_{2}\right)=\left\{\pi \in \mathcal{P}^{p}(X \times X) ;\right. \\
\left.\forall x \in X: \int_{X} \pi(x, y) d \mu(y)=\rho_{1}(x), \forall y \in Y: \int_{X} \pi(x, y) d \mu(x)=\rho_{2}(y)\right\} .
\end{aligned}
$$


$\pi$ contains information on how to transport $\rho_{1}$ onto $\rho_{2}$. $W_{d^{p}}\left(\rho_{1}, \rho_{2}\right)$ defines for $p \geq 1$ a distance between the PDFs $\rho_{1}$ and $\rho_{2}$, called " $p$-Wasserstein" distance for the metric $d$. Eq. (5) represents a linear programming problem (minimization of a linear functional with linear equality constraints). Despite the linearity, this problem can easilly become intractable because it implies a search in the space $\mathcal{P}^{p}(X \times X)$ that has large dimensionality in the seismic case. So, let us discuss more tractable reformulations.

Monge's problem (the original OT formulation) is a restriction of Kantorovich's problem to transference plans of the form $\pi(x, y)=\rho_{1}(x) \delta(y-T(x))$ where $T: X \rightarrow X$ is an invertible function, giving (Villani, 2008; Ambrosio and Gigli, 2009):

$$
\widehat{W}_{d^{p}}^{p}\left(\rho_{1}, \rho_{2}\right)=\min _{T \in \Sigma\left(\rho_{1}, \rho_{2}\right)} \int_{X} d^{p}(x, T(x)) \rho_{1}(x) d \mu(x),
$$

where the map $T$ is constrained to transport $\rho_{1}$ onto $\rho_{2}$ :

$$
\Sigma\left(\rho_{1}, \rho_{2}\right)=\left\{T: X \rightarrow X ; \forall x \in X: \rho_{1}\left[T^{-1}(x)\right]=\rho_{2}(x)\right\} .
$$

Monge's problem represents a restriction of Kantorovich's problem and cannot always be solved. However, it implies a search in the space of bijective functions defined on $X$, that is smaller than the Kantorovich search space. Despite this advantage, the constraint in eq. (8) is nonlinear and difficult to enforce as soon as the coordinate space is multiD, because it then involves resolving the Monge-Ampère equation (Villani, 2008). In practice, only the 1D coordinate space version has been used for industrial FWI applications, considering a trace-per-trace comparison, i.e. explicit correlations only in the time-direction. Used together with a transformation of each seismic trace into a PDF, here called a "PDF-transformation", this formulation proved to reduce the cycle-skipping issue in FWI (Engquist et al., 2016; Yang and Engquist, 2018; Yang et al., 2018; Wang and Wang, 2019). The limitations of this scheme are that in practice it does not allow the exploitation of multidimensionality in the coordinate space and that using "PDF-transformations" may lead to difficulties with field data (Métivier et al., 2018).

A simplification of the Kantorovich problem occurs when the metric $d$ is lower semi-continuous and $p=1$. This allows to switch to the Kantorovich-Rubinstein (KR) dual formulation, defined for $\rho_{1}$ and $\rho_{2}$ in the $\mathcal{P}^{1}(X)$ space by (Villani, 2008):

$$
W_{d}\left(\rho_{1}, \rho_{2}\right)=\max _{\varphi \in \operatorname{Lip}(d, 1)} \int_{X} \varphi(x)\left(\rho_{1}(x)-\rho_{2}(x)\right) d \mu(x),
$$

where we denote by $\operatorname{Lip}(d, \alpha)$ the set of $\alpha$-Lipschitz functions on $X$ with respect to the distance $d$ :

$$
\operatorname{Lip}(d, \alpha)=\left\{\varphi: X \rightarrow \mathbb{R} ; \max _{x \neq y} \frac{|\varphi(x)-\varphi(y)|}{d(x, y)} \leq \alpha, \varphi \in L^{1}\left(\left|\rho_{1}-\rho_{2}\right| d \mu\right)\right\} .
$$

$\varphi$ is constrained to be "slowly" varying, the absolute value of its "local slopes" being bounded by $\alpha=1$. The KR problem implies a search in the space of 1 -Lipschitz functions on $X$ (that must be integrable for the measure $\left|\rho_{1}-\rho_{2}\right| d \mu$, hence belong to $\left.L^{1}\left(\left|\rho_{1}-\rho_{2}\right| d \mu\right)\right)$. The Lipschitz constraint can be recast into linear constraints and leads to a linear programming problem that is numerically manageable even in the multiD case. Another nice feature of this formulation is that it is well defined not only for $\left(\rho_{1}, \rho_{2}\right) \in \mathcal{P}^{1}(X) \times \mathcal{P}^{1}(X)$, but also for any function $\left(f_{1}, f_{2}\right) \in L^{1}(X) \times L^{1}(X)$ provided they have the same "mass", i.e. $\int_{X} f_{1}(x) d \mu(x)=\int_{X} f_{2}(x) d \mu(x)$. This is still too constraining for seismic data (even if oscillating, seismic data cannot be considered to have approximately null mass within FWI, especially as many applications use the low frequencies and mutes). When $f_{1}$ and $f_{2}$ do not have same mass, the $\varphi$ inverted in eq. (10) becomes singular; a solution is to add a bounding constraint to $\varphi$ (Hanin, 1992; Lellmann et al., 2014). Introducing the " $\lambda$-bounded $\alpha$-Lipschitz" set

$$
\begin{aligned}
\operatorname{BLip}(d, \alpha, \lambda)=\{\varphi: X \rightarrow \mathbb{R} ; & \\
& \left.\max _{x \neq y} \frac{|\varphi(x)-\varphi(y)|}{d(x, y)} \leq \alpha,\|\varphi\|_{\infty} \leq \lambda, \varphi \in L^{1}\left(\left|f_{1}-f_{2}\right| d \mu\right)\right\},
\end{aligned}
$$

we now resolve for any function $\left(f_{1}, f_{2}\right) \in L^{1}(X) \times L^{1}(X)$

$$
\tilde{W}_{d}\left(f_{1}, f_{2}\right)=\max _{\varphi \in \operatorname{BLip}(d, 1, \lambda)} \int_{X} \varphi(x)\left(f_{1}(x)-f_{2}(x)\right) d \mu(x) .
$$


The absolute value of the "local slopes" of $\varphi$ is as above constrained to be bounded by $\alpha$, and in addition the absolute value of $\varphi$ is constrained to be thresholded by $\lambda$. $\tilde{W}_{d}$ still defines a distance between $f_{1}$ and $f_{2}$, induced by a norm called the "KR norm"; $\tilde{W}_{d}$ represents a generalization of $W_{d}$ and some relationship between both can be found in (Hanin, 1992; Villani, 2003; Lellmann et al., 2014). Solving for eq. (12) is still a linear programming problem.

Let us now consider coordinate space distances $d$ induced by a norm, i.e. $d(x, y)=\|x-y\|$; $(X, d)$ then becomes a Banach space (Rudin, 1991; Brezis, 2020). $d$ is typically induced by a Mahalanobis-like $L_{p}^{(X)}$ norm $(p \geq 1) \dagger$ :

$$
\|x\| \rightarrow\|x\|_{p}^{(X)}=\left(\frac{\left|x_{x l}\right|^{p}}{\sigma_{x l}^{p}}+\frac{\left|x_{i n l}\right|^{p}}{\sigma_{i n l}^{p}}+\frac{\left|x_{t}\right|^{p}}{\sigma_{t}^{p}}\right)^{1 / p} .
$$

The $\sigma$, with $\infty>1 / \sigma>0$, denote standard-deviation-like weights that can account for uncertainties and rescale the different physical dimensions between crossline and inline positions, and time. At the limit $p \rightarrow \infty$, the coordinate space "uniform norm" is defined by $\|x\|_{\infty}^{(X)}=$ $\max \left(\frac{\left|x_{x l}\right|}{\sigma_{x l}}, \frac{\left|x_{i n l}\right|}{\sigma_{i n l}}, \frac{\left|x_{t}\right|}{\sigma_{t}}\right)$.

The use of the KR norm within FWI has been proposed by Métivier et al. (2016a, $2016 \mathrm{~b}, 2016 \mathrm{c})$, with the specific choice $d \rightarrow\|.\|_{1}^{(X)}$ that they argued to be the most interesting computationally speaking; this choice will be implicit in the following. Theses authors proposed to use the SDMM algorithm to efficiently resolve the problem and underlined the many interesting properties of the KR norm. It allows the direct use of seismic data without application of a PDF-transformation as required by the approaches derived from Engquist and Froese (2014). Reduced sensitivity to cycle-skipping and amplitudes are satisfyingly achieved, mostly thanks to the 1-Lipschitz constraint that brings low frequencies and tends to equalize the amplitudes in the adjoint-source (Métivier et al., 2016b, 2016c; Poncet et al., 2018; Messud and Sedova, 2019). Fig. 1 illustrates that the KR norm global minimum valley outperforms the convexity of LSQ, giving a global minimum valley that is almost twice the width (even if the number of local minima, i.e. two, is the same). So far, it has been the only OT-based scheme implemented numerically in multiD in an industrial context, allowing a study of the advantage of taking into account the correlations of the events within common shot data; also, the corresponding tuning parameters are few and have a clear physical interpretation (Poncet et al., 2018; Messud and Sedova, 2019). Before detailing these points, we firstly clarify formal aspects related to the KR norm adjoint-source computation and precisely define the KR norm adjoint-source "texture", that represent the keys to better understanding the interest regarding the cycle-skipping problem.

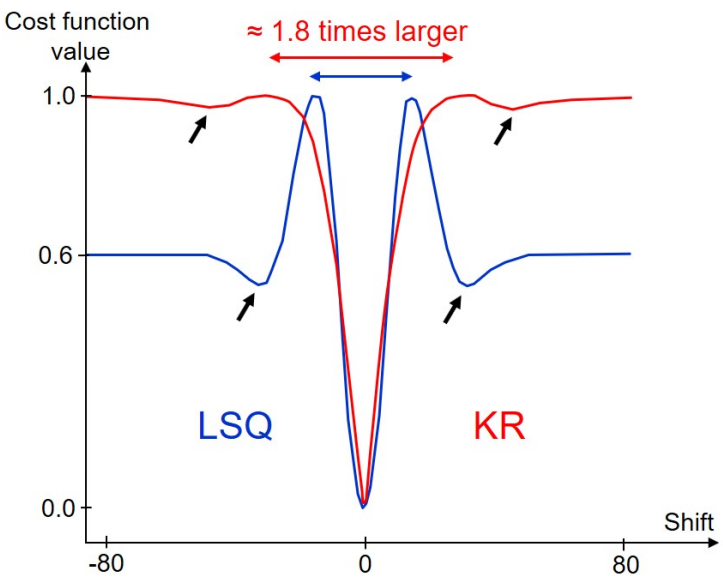

Figure 1. Comparison of the KR norm and LSQ cost function values for 2 shifted Ricker wavelets, the horizontal axis being the shift value. The global minimum valley is almost twice the width (even if the number of local minima is the same, two, highlighted by the black arrows), which is very interesting for FWI especially when dealing with the low frequencies.

$\dagger$ The coordinate space $L_{p}^{(X)}$-norm $\|x\|_{p}^{(X)}$, eq. (13), is not to be confused with the data space $L_{p}$-norm $\|f\|_{p}$, eq. (4), hence the superscript $(X)$ to make this explicit. 


\section{Analysis of the exact KR FWI adjoint-source}

\subsection{Form of the adjoint-source}

We consider a given shot data $f_{\text {obs }} \in L^{1}(X)$. Eq. (12) with $d \rightarrow\|.\|_{1}^{(X)}$ can be rewritten for all $f \in L^{1}(X)$

$$
\begin{aligned}
& \tilde{W}_{d}\left(f, f_{o b s}\right)=\int_{X} \varphi_{\max }[f](x)\left(f(x)-f_{o b s}(x)\right) d \mu(x) \\
& \varphi_{\max }[f]=\underset{\varphi \in \operatorname{BLip}\left(\|\cdot\|_{1}^{(X)}, 1, \lambda\right)}{\operatorname{argmax}} \int_{X} \varphi(x)\left(f(x)-f_{o b s}(x)\right) d \mu(x) .
\end{aligned}
$$

The KR adjoint-source, eq. (3), is thus defined by

$$
\frac{\partial \tilde{W}_{d}\left(f, f_{o b s}\right)}{\partial f(x)}=\varphi_{\max }[f](x)+\int_{X} \frac{\partial \varphi_{\max }[f]\left(x^{\prime}\right)}{\partial f(x)}\left(f\left(x^{\prime}\right)-f_{o b s}\left(x^{\prime}\right)\right) d \mu\left(x^{\prime}\right) .
$$

The second term, $\int_{X} \frac{\partial \varphi_{\max }[f]\left(x^{\prime}\right)}{\partial f(x)}\left(f\left(x^{\prime}\right)-f_{o b s}\left(x^{\prime}\right)\right) d \mu\left(x^{\prime}\right)$, is defined provided $\varphi_{\max }[f]$ is differentiable and has been neglected in Métivier et al. (2016a, 2016b, 2016c). In this section, we demonstrate that the differential $\partial \varphi_{\max }[f]\left(x^{\prime}\right) / \partial f(x)$ exists and can be neglected, fundamentally justifying the following form for the KR adjoint-source:

$$
\frac{\partial \tilde{W}_{d}\left(f, f_{o b s}\right)}{\partial f(x)}=\varphi_{\max }[f](x) .
$$

To achieve this goal, it is sufficient to prove

$$
\exists \gamma>0, \forall h \in L^{1}(X) \text { such as }\|h\|_{1}<\gamma: \quad \varphi_{\max }[f+h](x)=\varphi_{\max }[f](x),
$$

where the equality needs to hold almost everywhere "only" and

$$
\varphi_{\max }[f+h]=\underset{\varphi \in \operatorname{BLip}\left(\|\cdot\|_{1}^{(X)}, 1, \lambda\right)}{\operatorname{argmax}} \int_{X} \varphi(x)\left(f(x)-f_{o b s}(x)+h(x)\right) d \mu(x) .
$$

In other terms, if $\varphi_{\max }$ is invariant under any infinitesimal perturbations of the residual $f-f_{o b s}$, the differential $\partial \varphi_{\max }[f]\left(x^{\prime}\right) / \partial f(x)$ exists and is null. Intuitively, as we consider a maximization problem under constraints, eq. (17) would not be satisfied where the problem "hesitates" between different constraints saturations, so that infinitesimal perturbations of the residual may lead to "jumps" in the inverted $\varphi_{\max }$. Our following goal is to qualify subsets where this behavior can occur, i.e. where eq. (17) cannot be satisfied nor the differential defined.

To simplify, we firstly define a subset $X_{s u b} \subseteq X$ where it is the most pertinent to demonstrate eq. (17), called the subset that "matters".

\subsection{The subset that "matters"}

To simplify the following notations, we denote the residual by

$$
\Delta f=f-f_{o b s} .
$$

We denote by $X_{\text {null }}^{(\Delta f)}$ the subset of $X$ where the residual is infinitesimal:

$$
X_{\text {null }}^{(\Delta f)}=\{x \in X ; \forall \varepsilon>0:|\Delta f(x)| \leq \varepsilon\},
$$

and by $X_{\text {null }}^{(\Delta f)} \subseteq X_{\text {null }}^{(\Delta f)}$ where the spatial gradient of the residual is also infinitesimal:

$$
X_{\text {null }}^{(\Delta f)}=\left\{x \in X_{\text {null }}^{(\Delta f)} ; \forall \varepsilon>0:\left\|\frac{\partial}{\partial x} \Delta f(x)\right\|_{\infty}^{(X)} \leq \varepsilon\right\} .
$$

For realistic seismic data, $X_{\text {null' }}^{(\Delta f)}$ mostly corresponds to the area before the first arrival and the muted areas (that help the inversion to concentrate on the most relevant data like the diving waves). The blue areas in Fig. 2a give an illustration for a field data.

Fig. 2b gives an illustration on a simple synthetic trace. We observe that an additional contribution to the muted areas occurs in $X_{n u l l}^{(\Delta f)}$, represented by the brown areas, i.e. "deaf zones" where the residual stays very small while not on the edges. These contributions very rarely occur 
with realistic seismic data (unless specific subsurface structure or acquisition problems), as signal is usually permanently recorded between minimum and maximum experiment time.

Also, for any seismic data, $X_{\text {null }}^{(\Delta f)} \backslash X_{\text {null' }}^{(\Delta f)}$ (i.e. the complement of $X_{\text {null' }}^{(\Delta f)}$ in $X_{\text {null }}^{(\Delta f)}$ ) has a null Lebesgue measure in $X_{\text {null }}^{(\Delta f)}$ (thus in $X$ ) as it "just" represents where the oscillatory seismic signal passes through 0 . Corresponding contributions are partly represented by the dashed red curves in Fig. 2a, for a field 2D data, and by red points in Fig. 2b, for a simple synthetic 1D trace.

An important aspect with realistic FWI data and applications is that what happens in the $X_{\text {null }}^{(\Delta f)}$ subset has no real importance. Indeed, before being back-propagated, any FWI adjointsource is in practice muted (i.e. equalled to zero with possible rapid tapering) in the muted areas (or "blue areas"), and the rest of the $X_{\text {null }}^{(\Delta f)}$ contributions ("dashed red curves") has null measure. A consequence for $\mathrm{KR}$ adjoint-source considerations it that is sufficient to ensure that eq. (17) is satisfied $\forall x \in X \backslash X_{n u l l}^{(\Delta f)}$, the latter subset being called the subset that "matters" in the following $\dagger$. Note that this subset remains stable under infinitesimal perturbations of $\Delta f$.

(a) Field data receiver line (2D):

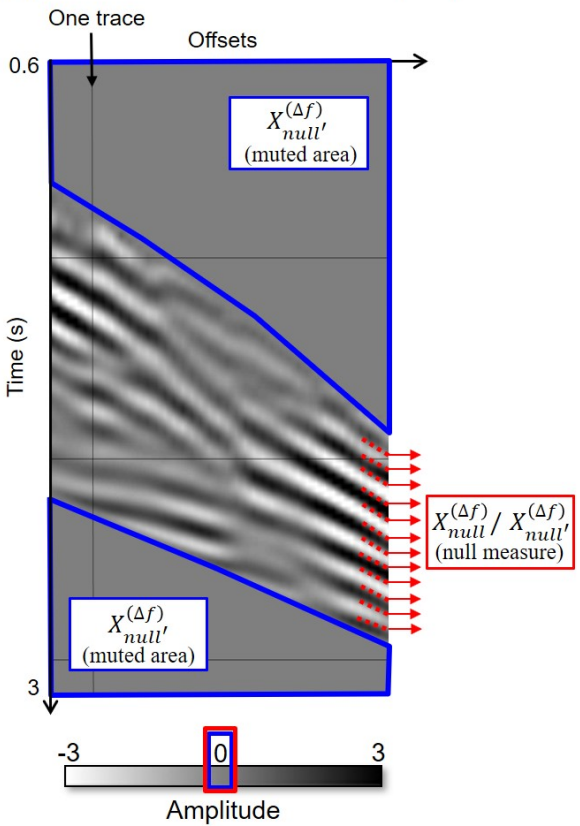

(b) Simple synthetic data trace with well separated events (1D):

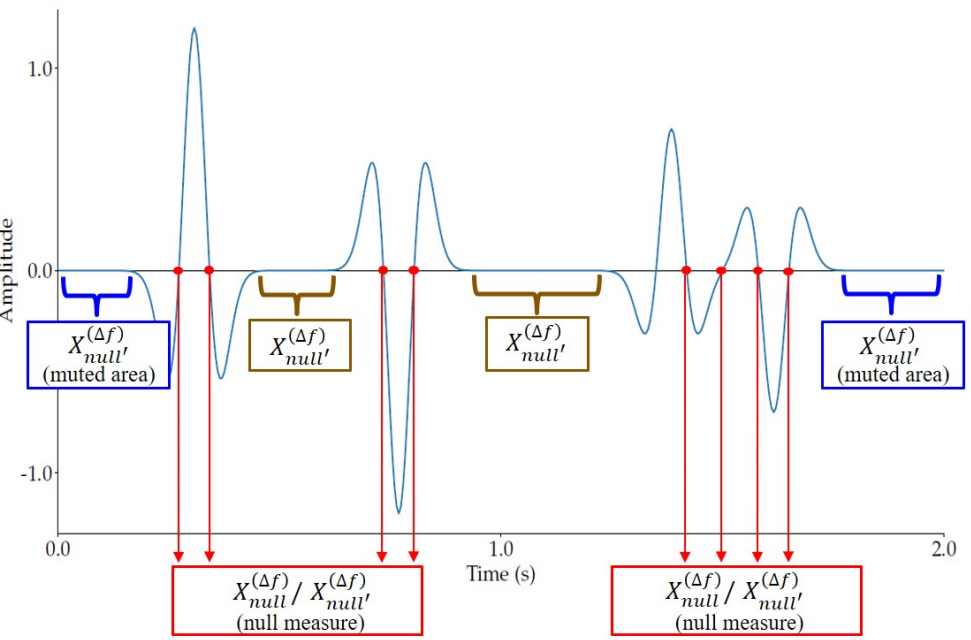

Figure 2. LSQ residual $\Delta f$ for (a) a field data receiver line (2D) and (b) a simple synthetic data trace (1D) (where $f_{o b s}$ contains two Ricker wavelets and $f$ contains the same Ricker wavelets but shifted). The various corresponding subsets of $X$ are explicited in each case. The various contributions to $X_{\text {null }}^{(\Delta f)}$ are represented by the blue and brown areas; the blue areas contribution corresponds to the muted (or edge) areas; the brown areas contribution corresponds to "deaf zones" which very rarely occur with realistic seismic data. The various contributions to $X_{\text {null }}^{(\Delta f)} \backslash X_{n u l l^{\prime}}^{(\Delta f)}$ have a null Lebesgue measure in $X$; they tend to be lines in the 2D case (partly represented in (a) by the dashed red curves) and points in the 1D case (indidated in (b) by the red points).

\subsection{Rewriting the 1-Lipschitz contraint}

To ease the next formal considerations, we rewrite the 1-Lipschitz constraint of eq. (11) in a form that is valid almost everywhere. We consider $d$ induced by the $L_{1}^{(X)}$ norm, i.e $p=1$ in eq. (13). Appendix A demonstrates that, for a differentiable $\varphi(x)$, the 1-Lipschitz constraint can be

$\dagger$ In the following, we will demonstrate formal results on the KR adjoint-source by reasoning in $X \backslash X_{n u l l}^{(\Delta f)}$. However, from tests on simple synthetic data containing "deaf zones", we will observe that the demonstrated results tend to remain true also in the "brown areas" contribution to $X_{n u l l}^{(\Delta f)}$. This is due to the strong continuity constraint in the KR maximization problem. 
rewritten

$$
\begin{aligned}
& \max _{x \neq y} \frac{|\varphi(x)-\varphi(y)|}{\|x-y\|_{1}^{(X)}}=\max _{x}\left\|\frac{\partial \varphi(x)}{\partial x}\right\|_{\infty}^{(X)} \leq 1, \\
& \quad \text { with } \quad\left\|\frac{\partial \varphi(x)}{\partial x}\right\|_{\infty}^{(X)}=\max \left(\sigma_{x l}\left|\frac{\partial \varphi(x)}{\partial x_{x l}}\right|, \sigma_{i n l}\left|\frac{\partial \varphi(x)}{\partial x_{i n l}}\right|, \sigma_{t}\left|\frac{\partial \varphi(x)}{\partial x_{t}}\right|\right) .
\end{aligned}
$$

Thus, when the $L_{1}^{(X)}$ norm is considered in the 1-Lipschitz constraint, the corresponding dual norm representation must be considered for the derivative $\partial \varphi(x) / \partial x$, i.e. the $L_{\infty}^{(X)}$ norm with non-inverted " $\sigma$ ".

As the 1-Lipschitz constraint implies strong continuity, thus differentiability of $\varphi(x)$ almost everywhere, and as our next considerations "just" need to be valid almost everywhere, we propose to consider $\partial \varphi(x) / \partial x$ in the following. Then, the 1-Lipschitz constraint implies almost everywhere in $X$ (which is implicit from now)

$$
\max \left(\sigma_{x l}\left|\frac{\partial \varphi(x)}{\partial x_{x l}}\right|, \sigma_{i n l}\left|\frac{\partial \varphi(x)}{\partial x_{i n l}}\right|, \sigma_{t}\left|\frac{\partial \varphi(x)}{\partial x_{t}}\right|\right) \leq 1 .
$$

\subsection{A piecewise linear function}

In this section, we formally study the "shape" of $\varphi_{\max }$. This will allow us to prove eq. (17) but also to draw interesting lessons like rigorously defining a "texture" for the KR adjoint-source. We start by simplified cases before considering the "full" case.

\subsubsection{Simplified case (thresholding constraint only)}

We firstly consider only the thresholding constraint $\|\varphi\|_{\infty} \leq \lambda$ in eqs. (11) and (14), i.e. removing the Lipschitz constraint. Appendix B demonstrates that the solution of the problem in eq. (14) is then

$$
\begin{aligned}
x \in X \backslash X_{\text {null }}^{(\Delta f)} \text { with } \Delta f(x)>0: & \varphi_{\max }(x)=\lambda \\
\Delta f(x)<0: & \varphi_{\max }(x)=-\lambda .
\end{aligned}
$$

This result defines $\varphi_{\max }$ in $X \backslash X_{\text {null }}^{(\Delta f)}$, where it clearly remains stable under infinitesimal perturbations of $\Delta f$. We thus can deduce eq. (17) in the subset that matters $X \backslash X_{n u l l}^{(\Delta f)}$.

Note that the thresholding constraint saturates everywhere in $X \backslash X_{n u l l}^{(\Delta f)}$, see Fig. 3 for an illustration on a simple synthetic trace (shifted Rickers). Note also that the solution of eq. (24) can be rewritten

$$
x \in X \backslash X_{\text {null }}^{(\Delta f)}: \varphi_{\max }[f](x)=\lambda \operatorname{sign}\left(f(x)-f_{\text {obs }}(x)\right)=\lambda \frac{\partial}{\partial f(x)}\left\|f-f_{\text {obs }}\right\|_{1} .
$$

This simplified case is thus equivalent to the $L_{1}$ norm cost function case, up to a multiplicative factor $\lambda$ that has no impact because of the line-search that will recompute the global scaling.

\subsubsection{Intermediary case (1-Lipschitz constraint only)}

We now consider only the 1-Lipschitz constraint in eqs. (11) and (14), i.e. remove the thresholding constraint, which implies that the residual satisfies

$$
\int_{X} \Delta f(x) d \mu(x)=0
$$

to avoid singularities, remind $\S 2.2$.

Considering firstly a $1 \mathrm{D}$ coordinate space $X=[0, T]$, in the time direction, eq. (23) implies $\forall x_{t} \in X: \sigma_{t}\left|\frac{\partial \varphi\left(x_{t}\right)}{\partial x_{t}}\right| \leq 1$. Appendix B demonstrates that the solution of the problem in eq. (14) is then

$$
\begin{aligned}
x_{t} \in X \backslash X_{\text {null }}^{\left(\Delta F_{\beta}\right)} \text { with } \Delta F_{\beta}\left(x_{t}\right)<0: & \beta^{+}\left(x_{t}\right)=-\Delta F_{\beta}\left(x_{t}\right), \beta^{-}\left(x_{t}\right)=0, \sigma_{t} \frac{\partial \varphi_{\max }\left(x_{t}\right)}{\partial x_{t}}=1 \\
\Delta F_{\beta}\left(x_{t}\right)>0: & \beta^{-}\left(x_{t}\right)=\Delta F_{\beta}\left(x_{t}\right), \beta^{+}\left(x_{t}\right)=0, \sigma_{t} \frac{\partial \varphi_{\max }\left(x_{t}\right)}{\partial x_{t}}=-1,
\end{aligned}
$$


where

$$
\Delta F_{\beta}\left(x_{t}\right)=\int_{0}^{x_{t}} \Delta f(x) d \mu(x)-\left(\beta^{+}(0)-\beta^{-}(0)\right)
$$

defines the set $X_{\text {null }}^{\left(\Delta F_{\beta}\right)}$ through eq. (20). Eq. (27) contributes to define $\varphi_{\max }$ in $X \backslash X_{\text {null }}^{\left(\Delta F_{\beta}\right)}$ but implies the resolution of a self-consistent problem. Indeed, $\beta^{+}, \beta^{-}$and $\varphi_{\max }$ are coupled through $\Delta F_{\beta}$, but also through the necessary continuity of $\varphi_{\max }$ in the whole set $X$, i.e. also in $X_{\text {null }}^{\left(\Delta F_{\beta}\right)}$.

So, even if these equations would not lead to the most practical numerical scheme, they allow us to draw interesting formal conclusions:

- Eq. (27) clearly remains stable under infinitesimal perturbations of $\Delta F_{\beta}$, eq. (28), the latter remaining stable under infinitesimal perturbation of $\Delta f$. This implies that eq. (17) is satisfied in $X \backslash X_{\text {null }}^{\left(\Delta F_{\beta}\right)}$, and thus in $X \backslash X_{\text {null }}^{(\Delta f)}$ (as $X \backslash X_{\text {null }}^{\left(\Delta F_{\beta}\right)}$ tends to be "larger" for seismic data). Appendix B gives further analysis.

- The derivative constraint saturates almost everywhere in $X \backslash X_{\text {null }}^{\left(\Delta F_{\beta}\right)}$, thus in $X \backslash X_{\text {null }}^{(\Delta f)}$. But, in most cases, the derivative of $\varphi_{\max }$ will also tend to saturate in $X_{\text {null }}^{(\Delta f)}$, which is illustrated in Fig. 3 on a simple synthetic trace (built from shifted Ricker wavelets). Indeed, even if $X_{\text {null }}^{(\Delta f)}$ does not play an explicit role in eq. (27), it plays a role through the necessary continuity constraint on $\varphi_{\max }$ and the maximization of $\int_{X} \varphi\left(x_{t}\right) \Delta f\left(x_{t}\right) d \mu\left(x_{t}\right)$. This will tend to produce a piecewise linear shape for $\varphi_{\max }$ in the whole $X$, as visible in Fig. 3 .

- Fig. 3 also shows how the Lischitz constraint saturation leads to a reduction in amplitude dynamics in $\varphi_{\max }$ compared to $\Delta f$.

- The "oscillations" of $\varphi_{\max }$ are driven by $\Delta F_{\beta}$ through eq. (27), thus among others by the integral of $\Delta f$, eq. (28). This should give $\varphi_{\max }$ a lower frequency content than $\Delta f$, which can be deduced from Fig. 3 and is quantified in Fig. 4 . We observe that $\varphi_{\max }$ has its spectrum shifted towards the low frequencies, beyond the low frequencies of the integrated LSQ residual.

- The argmax (i.e. $\varphi_{\max }$ ) of the considered problem is not unique (Villani, 2008). In particular, any

$$
\varphi_{\max }^{(c)}(x)=\varphi_{\max }(x)+c, \quad \forall c \in \mathbb{R}
$$

clearly remains a solution of eqs. (14) and (10), or of eqs. (27) and (28), because eq. (26) must be satisfied. The constant $c$ does not change the value of the max, i.e. of $\tilde{W}_{d}$, but only the argmax. In schemes that use the argmax (like FWI where the argmax is interpreted as the adjoint-source), the constant may play a role, possibly producing a low frequency smearing.

\subsubsection{Full case and KR "texture"}

As demonstrated in Appendix B, considering both the thresholding and the 1-Lipschitz constraints in eqs. (11) and (14) and still a $1 \mathrm{D}$ coordinate space $X=[0, T]$, we obtain for $\varphi_{\max }$ :

$$
\begin{gathered}
x_{t} \in X \backslash X_{\text {null }}^{\left(\Delta F_{\beta, \alpha}\right)} \text { with } \Delta F_{\beta, \alpha}\left(x_{t}\right)<0: \quad \beta^{+}\left(x_{t}\right)=-\Delta F_{\beta, \alpha}\left(x_{t}\right), \beta^{-}\left(x_{t}\right)=0, \sigma_{t} \frac{\partial \varphi_{\max }\left(x_{t}\right)}{\partial x_{t}}=1 \\
\Delta F_{\beta, \alpha}\left(x_{t}\right)>0: \quad \beta^{-}\left(x_{t}\right)=\Delta F_{\beta, \alpha}\left(x_{t}\right), \beta^{+}\left(x_{t}\right)=0, \sigma_{t} \frac{\partial \varphi_{\max }\left(x_{t}\right)}{\partial x_{t}}=-1 \\
x_{t} \in X \backslash X_{\text {null }}^{\left(\Delta f_{\beta}\right)} \text { with } \begin{aligned}
& \Delta f_{\beta}\left(x_{t}\right)>0: \alpha^{+}\left(x_{t}\right)=\Delta f_{\beta}\left(x_{t}\right), \alpha^{-}\left(x_{t}\right)=0, \varphi_{\max }\left(x_{t}\right)=\lambda \\
& \Delta f_{\beta}\left(x_{t}\right)<0: \quad \alpha^{-}\left(x_{t}\right)=-\Delta f_{\beta}\left(x_{t}\right), \alpha^{+}\left(x_{t}\right)=0, \varphi_{\max }\left(x_{t}\right)=-\lambda,
\end{aligned}
\end{gathered}
$$

where $\left(\Delta F_{\beta}\right.$ being defined by eq. (28))

$$
\begin{aligned}
& \Delta F_{\beta, \alpha}\left(x_{t}\right)=\Delta F_{\beta}\left(x_{t}\right)-\int_{0}^{x_{t}} d x\left(\alpha^{+}(x)-\alpha^{-}(x)\right) d \mu(x) \\
& \Delta f_{\beta}\left(x_{t}\right)=\Delta f\left(x_{t}\right)+\frac{\partial \beta^{+}\left(x_{t}\right)}{\partial x_{t}}-\frac{\partial \beta^{-}\left(x_{t}\right)}{\partial x_{t}} .
\end{aligned}
$$

The two group of equations in eq. (30) define $\varphi_{\max }$ quite similarly than in eq. (27), with an additional explicit coupling. An implicit coupling is also imposed through the necessary (strong) 
continuity of $\varphi_{\max }$ and the fact that none of the constraints can saturate simultaneously, i.e. (see Appendix B)

$$
X_{\text {null }}=X_{\text {null }}^{\left(\Delta f_{\beta}\right)} \cup X_{\text {null }}^{\left(\Delta F_{\beta, \alpha}\right)} \quad \text { and } \quad \varnothing=X_{\text {null }}^{\left(\Delta f_{\beta}\right)} \cap X_{\text {null }}^{\left(\Delta F_{\beta, \alpha}\right)} .
$$

All this allows us to draw similar lessons than these underlined above:

- We deduce that eq. (17) is satisfied in the subset that matters $X \backslash X_{\text {null }}^{(\Delta f)}$, concluding the proof that the KR adjoint-source is given by eq. (16).

- The 1-Lipschitz constraint or the thresholding constraint tend to saturate (not simultaneously) almost everywhere in $X \backslash X_{\text {null }}^{(\Delta f)}$ and also in most of $X_{\text {null }}^{(\Delta f)}$. This will tend to produce a piecewise linear shape for $\varphi_{\max }$ in the 1D case, as illustrated in Fig. 5. Note that, in the multiD case, the piecewise linear shape becomes approximate. Indeed, at least one component of the 1-Lipschitz constraint then needs to saturate at a given position (mostly the time direction in our implementation), but nothing constrains many components to saturate simultaneously in the general case. This will be further discussed in the next section.

- Visible in Fig. 5 and for the reason mentioned in $\$ 3.4 .2$, the KR adjoint-source $\varphi_{\max }$ has a lower frequency content than the LSQ residual.

- Fig. 5 also shows how the constraints saturation lead to a reduced amplitude dynamics in $\varphi_{\max }$ compared to $\Delta f$, the thresholding constraint parameter $\lambda$ allowing further control of these dynamics (in the extreme case of a very small $\lambda$, the KR adjoint-source would become equivalent to the $L_{1}$ adjoint-source up to a global proportionality constant).

- These three previous items (piecewise linearity, lower frequency content, reduced amplitude dynamics) represent what we call KR "texture" for the adjoint-source in the following. It is favorable for reducing the sensitivity of FWI to cycle-skipping and amplitudes.

- The thresholding constraint, making eq. (26) useless, limits the additive constant (or null frequency) issue discussed in §3.4.2. However, in a situation where the chosen $\lambda$ value does not provide enough constraint and eq. (26) approximately holds, the additive constant issue can still exist, that could produce a low frequency smearing in the FWI adjoint-source. A possible effect will be discussed later in $§ 4.7$.

- Eq. (17) can remain valid for non-infinitesimal perturbations $h$, hence the often mentioned robustness of the KR adjoint-source to noise. Fig. 6 gives an illustration for a quite strong uniform noise. We observe that the KR adjoint-source is perturbed by the presence of noise mostly in $X_{\text {null }}^{(\Delta f)}$, that does not really matter, and tends to remain very stable in the subset that matters $X \backslash X_{\text {null }}^{(\Delta f)}$.

- Finally, we mention that the approximate piecewise linear shape for $\varphi_{\max }$ in the multiD case is due to the use of a distance $d$ induced by the $L_{1}^{(X)}$ norm in the 1-Lipschitz constraint of eq. (11), that leads to the constraint in eq. (23). If a $L_{p}^{(X)}$ norm with $\left.p \in\right] 1, \infty[$ was used instead, the constraint in eq. (23) would have to be replaced by the following constraint (as demonstrated in Appendix A)

$$
\left(\sigma_{x l}^{q}\left|\frac{\partial \varphi(x)}{\partial x_{x l}}\right|^{q}+\sigma_{i n l}^{q}\left|\frac{\partial \varphi(x)}{\partial x_{i n l}}\right|^{q}+\sigma_{t}^{q}\left|\frac{\partial \varphi(x)}{\partial x_{t}}\right|^{q}\right)^{1 / q} \leq 1 \quad \text { with } \quad 1 / p+1 / q=1 .
$$

The saturation of this constraint would not lead to approximate piecewise linearity in the general multiD case, as the derivatives in the various coordinate space directions would be strongly coupled. 


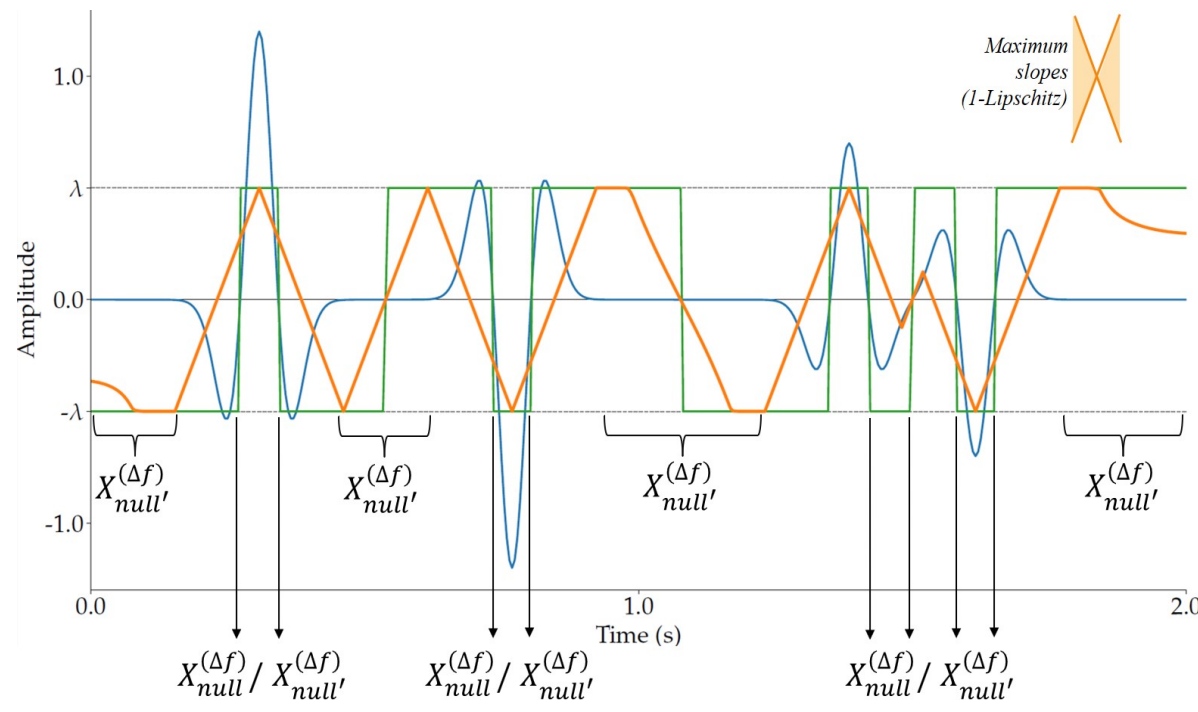

Figure 3. 1D coordinate space $X=[0, T]$ (time direction). Comparison of adjoint-sources for a simple synthetic trace, where $f_{\text {obs }}$ contains two Ricker wavelets (all with peak frequency at 6 $\mathrm{Hz}$ ) and $f$ contains the same Ricker wavelets but shifted. In blue, the LSQ residual. In green, the $L_{1}$ adjoint-source. In orange, the KR adjoint-source, which is computed using the default linear cone iterative solver of the open source sccvxopt library (Andersen et al., 2013), ran until convergence. At the bottom the various corresponding subsets of $X$. The KR adjoint-source tends to saturate the (1-Lipschitz or thersholding) constraints in most of $X$ and especially in $X \backslash X_{\text {null }}^{(\Delta f)}$.

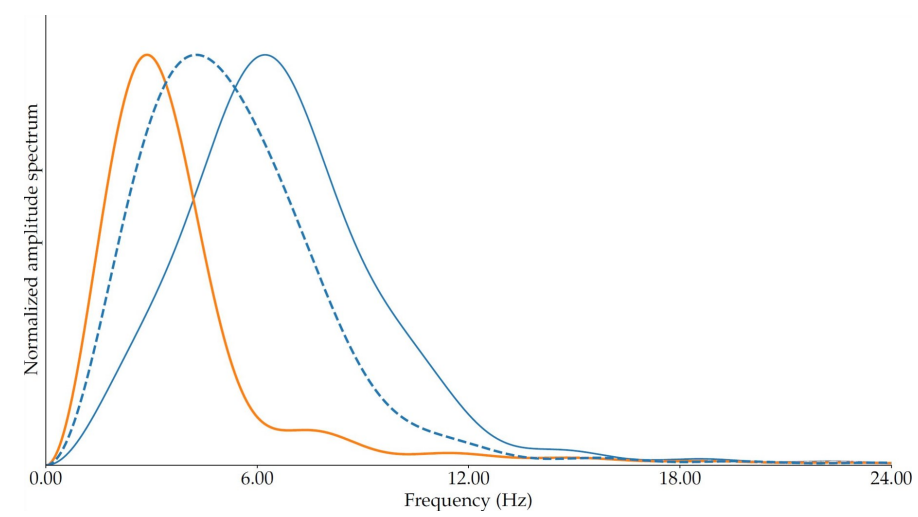

Figure 4. Comparison of the amplitude spectra of different adjoint-sources corresponding to the same setting as Fig. 3. In blue, the LSQ residual (same spectrum as the one of a Ricker wavelet whose peak frequency is at $6 \mathrm{~Hz}$ ). In dashed blue, the spectrum of the integrated LSQ residual. In orange, the KR adjoint-source. The KR adjoint-source has its spectrum shifted towards the low frequencies, beyond the low frequencies of the integrated LSQ residual. This is favorable to help overcoming cycle-skipping within FWI (up to the extent of the low frequencies present in the forward propagated source wavelet). 


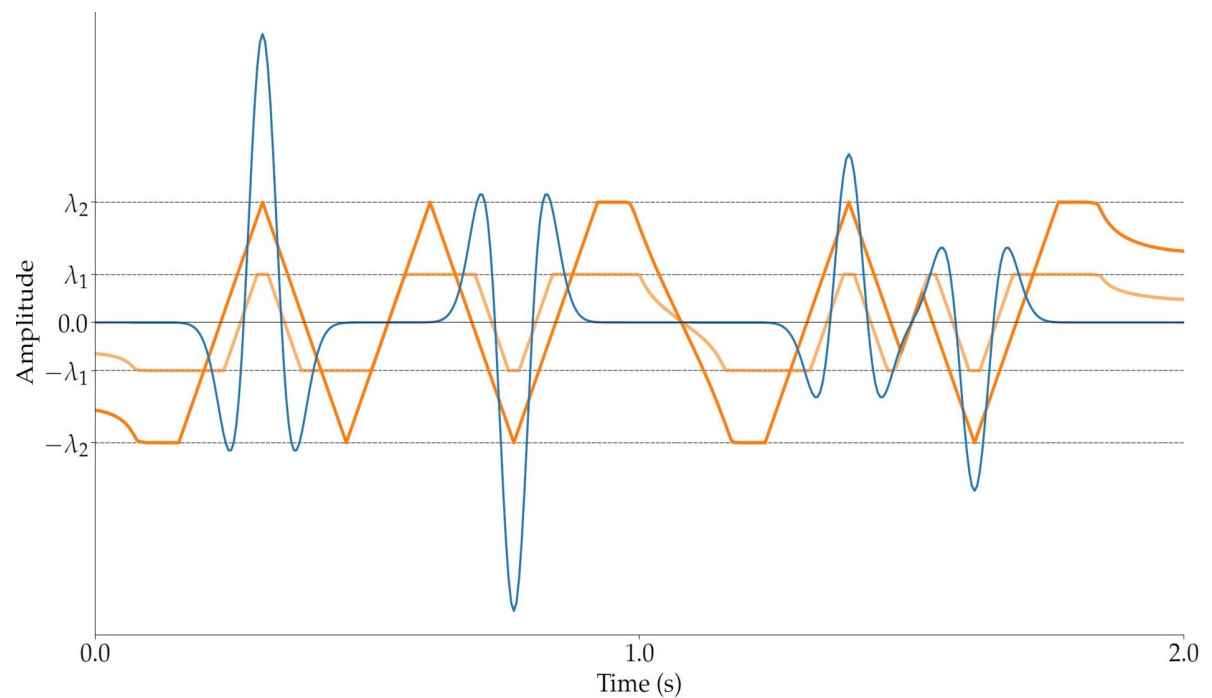

Figure 5. Comparison of adjoint-sources for the same setting as Fig. 3. In blue, the LSQ residual. In orange, the KR adjoint-sources corresponding to bound $\lambda_{2}$ (dark orange) and $\lambda_{1}$ $\left(<\lambda_{2}\right.$, light orange). Smaller $\lambda$ values make the thresholding constraint saturate more, producing more clipping of the adjoint-source.

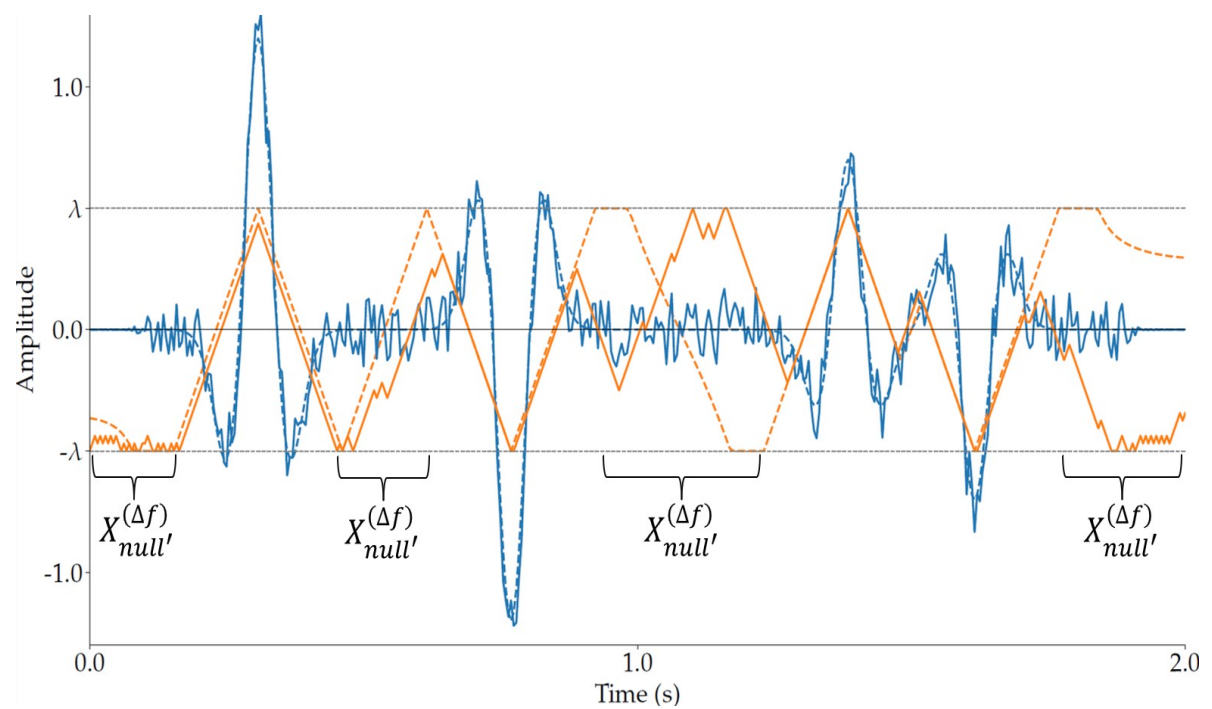

Figure 6. Comparison of adjoint-sources for a similar setting than in Fig. 3, with additional uniform noise. In blue, the LSQ residual to which uniform noise has been added (the LSQ residual without noise is blue dashed). The level of noise corresponds to $30 \%$ of the maximum amplitude of the LSQ residual. In orange, the KR adjoint-source inverted from noisy LSQ residual (the $\mathrm{KR}$ adjoint-source without noise is orange dashed). The KR adjoint-source is perturbed by the presence of noise mostly in $X_{n u l l}^{(\Delta f)}$, that does not really matter. 


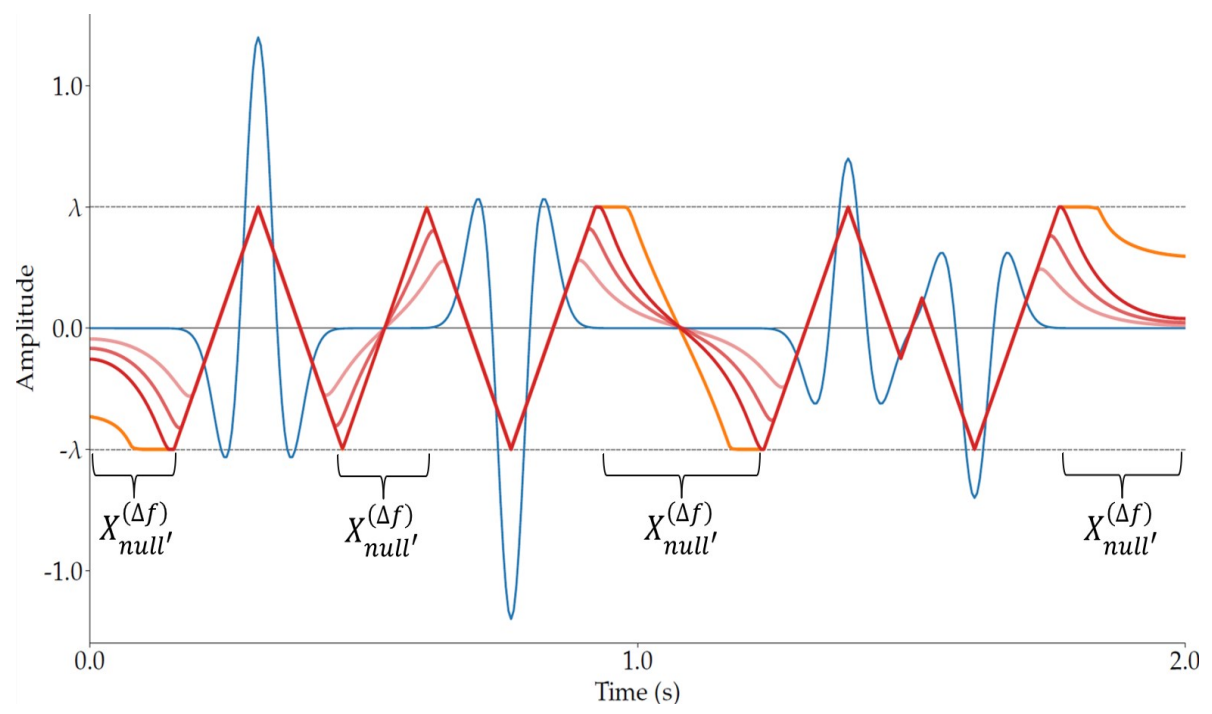

Figure 7. Comparison of adjoint-sources for a similar setting than in Fig. 3. In blue, the LSQ residual. In orange, the exact KR adjoint-source. In various reds, the KR adjoint-source approximated using SDMM with 50 (light red), 500 and 5000 (dark red) iterations. The various SDMM KR adjoint-sources differ from the exact KR adjoint-source mostly on $X_{\text {null }}^{(\Delta f)}$, that does not really matter.

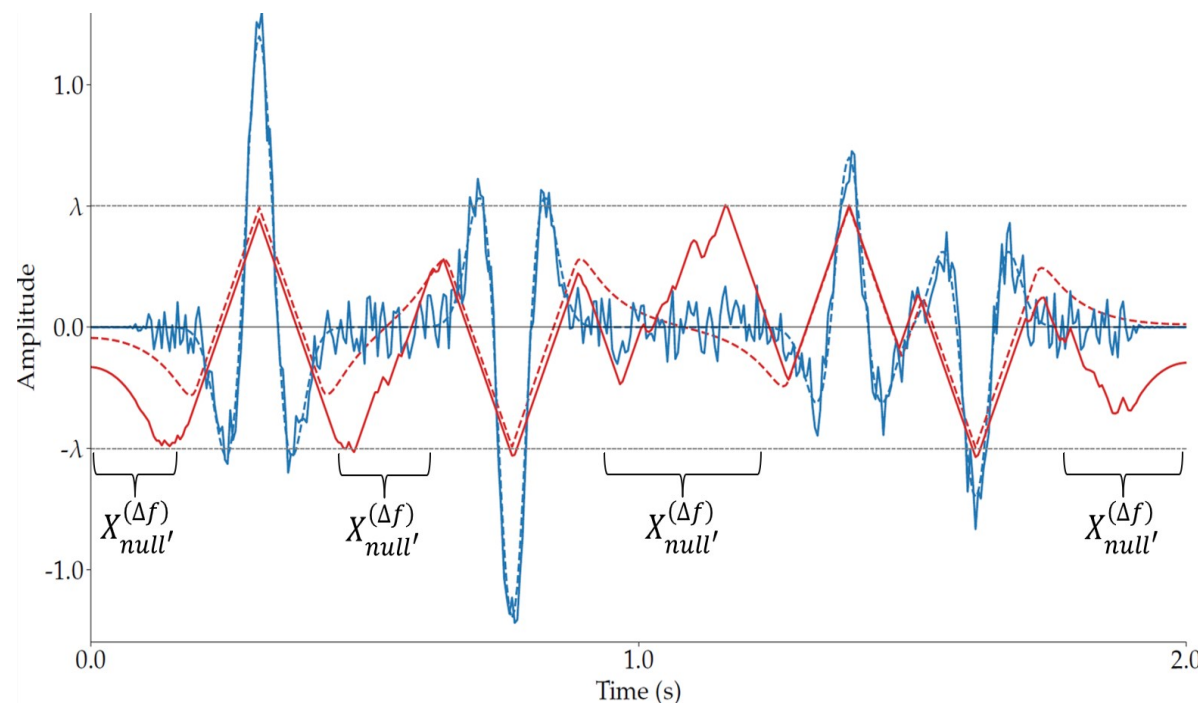

Figure 8. Comparison of adjoint-sources for the same setting as Fig. 6. In blue, the noisy LSQ residual (the LSQ residual without noise is dashed). In red, the SDMM (50 iterations) KR adjoint-source inverted from the inverted the noisy LSQ residual (the corresponding SDMM KR adjoint-source without noise is dashed). The SDMM KR adjoint-source is perturbed by the presence of noise mostly in $X_{\text {null }}^{(\Delta f)}$, that does not really matter. Note that it seems a little less perturbed by the noise (in $X_{\text {null }}^{(\Delta f)}$ ) than the exact KR adjoint-source of Fig. 6 .

\section{Practical aspects}

\subsection{SDMM approach and illustration on a simple synthetic trace}

Up to now, we have considered an exact resolution of the KR problem. In this section, we underline the advantages of using an approximate resolution method, the SDMM convex optimization with a Laplace solver (Métivier et al., 2016b).

$N \geq 0$ denotes the number of SDMM inner iterations. In our implementation, we start iterating taking the LSQ residual $\Delta f$ as the initial function for $\varphi$. Thus, for $N=0$, the scheme 
is equivalent to LSQ. Increasing $N$ will "add" to the adjoint-source more and more of the KR texture defined in \$3.4.3, linearly increasing the computational cost. An exact resolution of the KR problem would be obtained using a very large $N$, which would be too costly for large scale applications and, mostly, unecessary. Fig. 7 shows the exact KR adjoint-source and the ones approximated by SDMM $(N=50,500$ and 5000) on a simple synthetic trace (shifted Ricker wavelets). Interestingly, all results are very similar in the subset that matters $X \backslash X_{\text {null }}^{(\Delta f)}$ and differ mostly on $X_{\text {null }}^{(\Delta f)}$ where SDMM takes more iterations to converge. As what happens in $X_{\text {null }}^{(\Delta f)}$ does not really matter in practice, the adjoint-source obtained with the smaller $N$ value, i.e. $N=50$, seems sufficient. We observe that the KR texture (piecewise linearity, lower frequency content, reduced amplitude dynamics) is mostly preserved even for $N=50$.

The $N$ parameter thus has a clear meaning, controlling the desired amount of KR texture added to the LSQ residual, allowing us to consider the KR norm as a hybrid misfit that mixes OT with the LSQ cost function. This flexibility is a strong point for industrial applications. For instance, at the first FWI iteration, more KR texture can be added by increasing $N$ and, as FWI converges, $N$ may be reduced to tend to LSQ.

What about SDMM robustness to noise? Fig. 8 illustrates the KR adjoint-source inverted by SDMM with $N=50$, for a noisy LSQ residual. We observe that the KR adjoint-source is perturbed by the presence of noise mostly in $X_{\text {null }}^{(\Delta f)}$, that does not really matter, and tends to remain very stable in $X \backslash X_{\text {null }}^{(\Delta f)}$, which is satisfying. Interestingly, the SDMM KR adjoint-source seems a little less perturbed by the noise in $X_{\text {null }}^{(\Delta f)}$ than the exact KR adjoint-source, comparing Figs. 6 and 8.

This section justifies the use of SDMM to compute the KR adjoint-source far beyond computational cost considerations only. In practice, our optimizations and tuning of SDMM allow ta satisfying result to be reached for $N=20$ to 60 .

\subsection{Data space "splitting", and $1 D$ and $2 D K R$}

We mentioned that eq. (14) can be resolved considering different effective dimensionalities for the coordinate space $X$. In our implementation, we considered two cases:

(i) Each trace within a shot independently. The coordinate space is not anymore multiD but $1 D$ (that is still "much more" than $L_{p}$ norms whose coordinate space dimensionality is 0 as they are computed point-wise), the effective $X$ being $[0, T]$. Only correlations in the time direction can be accounted for and we call this scheme $1 \mathrm{D}$ KR in the following.

(ii) Group of traces in one spatial direction, the best sampled or less aliased one, thus an effective dimensionality of 2 . Correlations in the chosen spatial and time directions can be accounted for and we call this scheme $2 \mathrm{D} \mathrm{KR}$ in the following. For instance in the marine case, we consider each receiver line within a shot independently, the effective $X$ being $\left[H_{i n l}^{\min }, H_{i n l}^{\max }\right] \times[0, T]$.

Fig. 9 illustrates this data space "splitting" for 1D and 2D KR. 


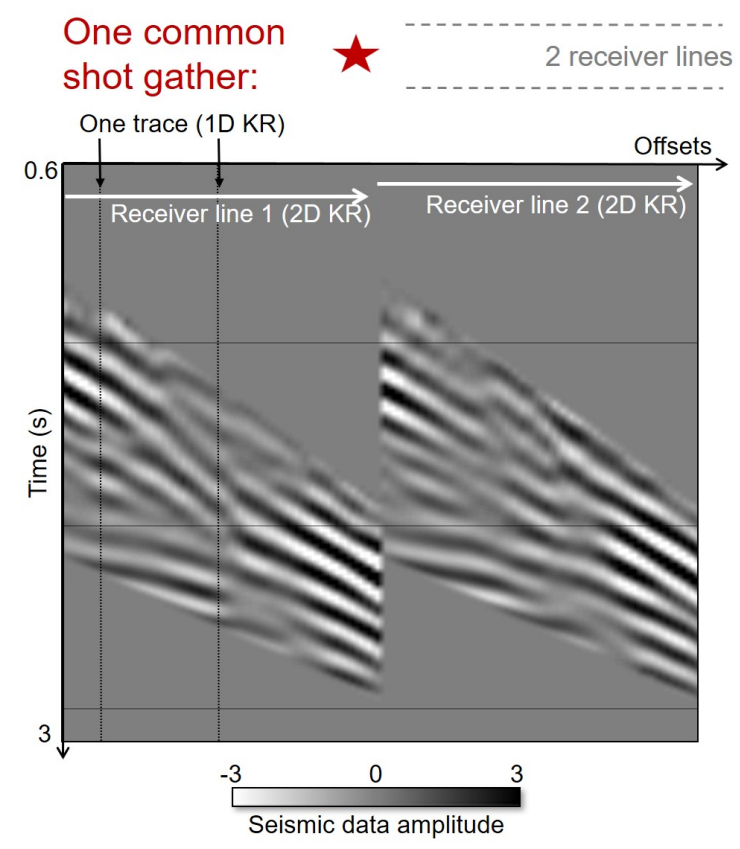

Figure 9. Marine field data set corresponding to two receiver lines within a common shot gather. LSQ residual at $6 \mathrm{~Hz}$, muted for the FWI, that represents the initial adjoint-source for SDMM iterations. 2D KR considers each receiver line independently and 1D KR considers each trace independently. Modified from Poncet et al. (2018) (see the article for more details).

\subsection{SDMM KR illustrated on common shot data}

We firstly consider a 2D common shot data modelled in the Marmousi 2 model (Martin et al., 2006). The source is a Ricker wavelet with peak frequency at $6 \mathrm{~Hz}$ and the data has been low-pass filtered below $3 \mathrm{~Hz}$ to be more realistic. Fig. 10 illustrates the effect of the SDMM number of iterations $N$. The results are shown with a smooth high-cut filter above $4 \mathrm{~Hz}$ to make it clearer to see the wiggle traces. We can observe how more and more KR texture is added to the KR adjoint-source as $N$ increases. The wiggles highlight how each KR adjoint-source trace tends to becomes more piecewise linear, especially in the time direction that matters the most to overcome cycle-skipping.

Some artefacts can be observed in Fig. 10 at large offsets as the amplitudes tend to be larger and the traces are further from null average. However, these artefacts remain very localized. We can observe that using 2D KR diminishes the artefacts and improves the adjoint-source continuity compared to using 1D KR. We will detail the corresponding specifics of $2 \mathrm{D}$ KR further in $\S 4.6$.

Fig. 10 also illustrates how the KR adjoint-source amplitude dynamics are reduced as $N$ increases, and lets guess that the low frequency content is reinforced. This is confirmed by Fig. 11 (high-cut at $10 \mathrm{~Hz}$ instead of $4 \mathrm{~Hz}$ to make frequency spectra visualization clearer), even for the smaller $N=20$ value.

Secondly, we consider a field 3D common shot data. Fig. 12 compares a LSQ residual with the corresponding 2D KR adjoint-source computed by SDMM, with a mute applied as usually done for FWI. Again, the 2D KR adjoint-source has more low frequencies and a reduced amplitude dynamics.

In this section, the KR texture for the adjoint-source computed by SDMM has been illustrated on shot data and in the 1D and multiD cases. This texture represents a key to mitigate the cycle-skipping and reduce the sensitivity to the amplitudes within FWI, enhancing the kinematic information present in the adjoint-source (like a smart processing of the LSQ residual). To make this point explicit, the KR texture is sometimes called "skeleton-like" texture. 

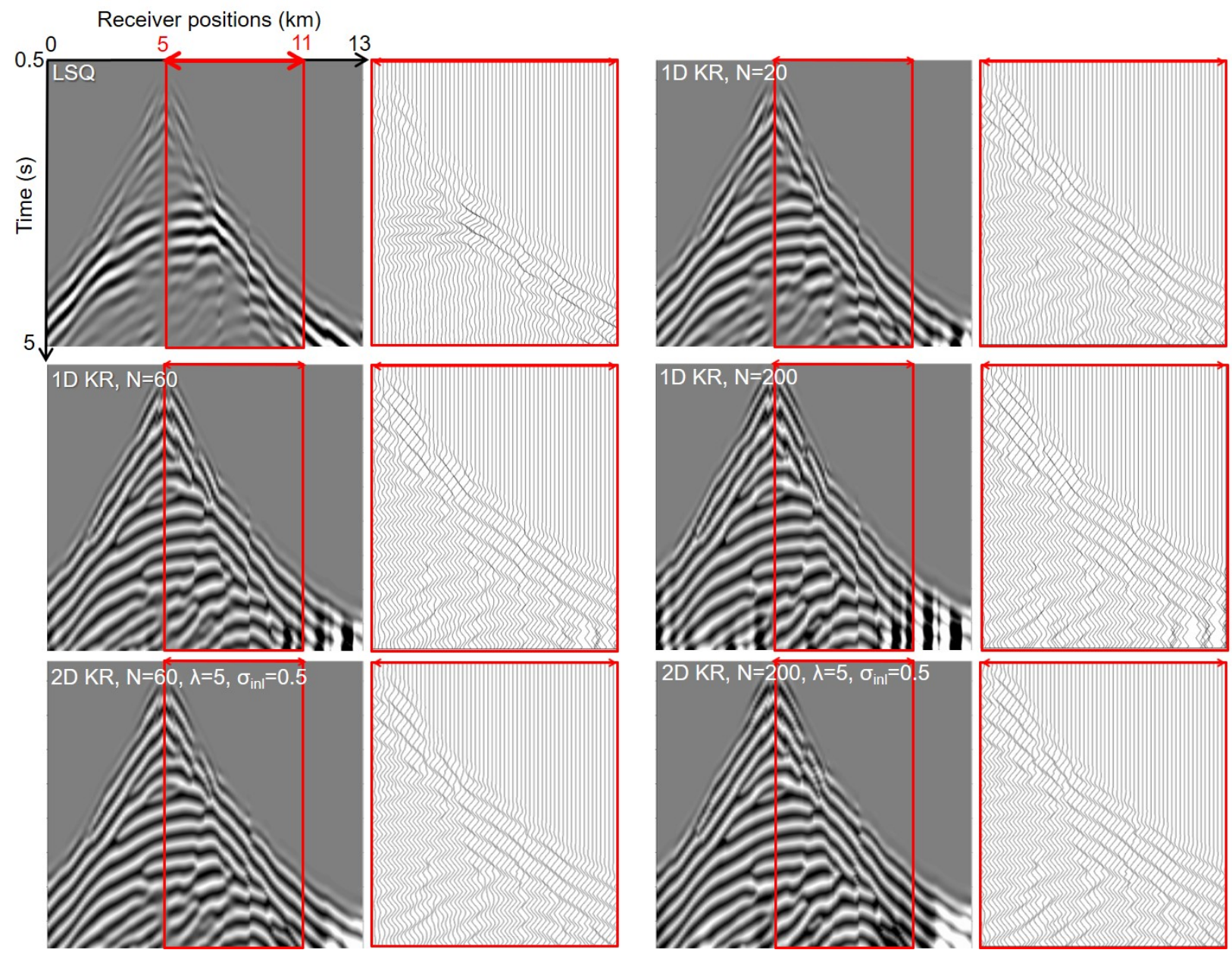

Figure 10. Marmousi 2 data set (Martin et al., 2006) (Ricker wavelet with peack frequency at 6 $\mathrm{Hz}$ and frequencies below $3 \mathrm{~Hz}$ muted). A smooth high-cut filter above $4 \mathrm{~Hz}$ has been applied to make it easier to see the wiggle traces. LSQ residual, and 1D and 2D KR adjoint-sources (effect of the number of iterations $N$ ) are shown, for one common shot gather. 

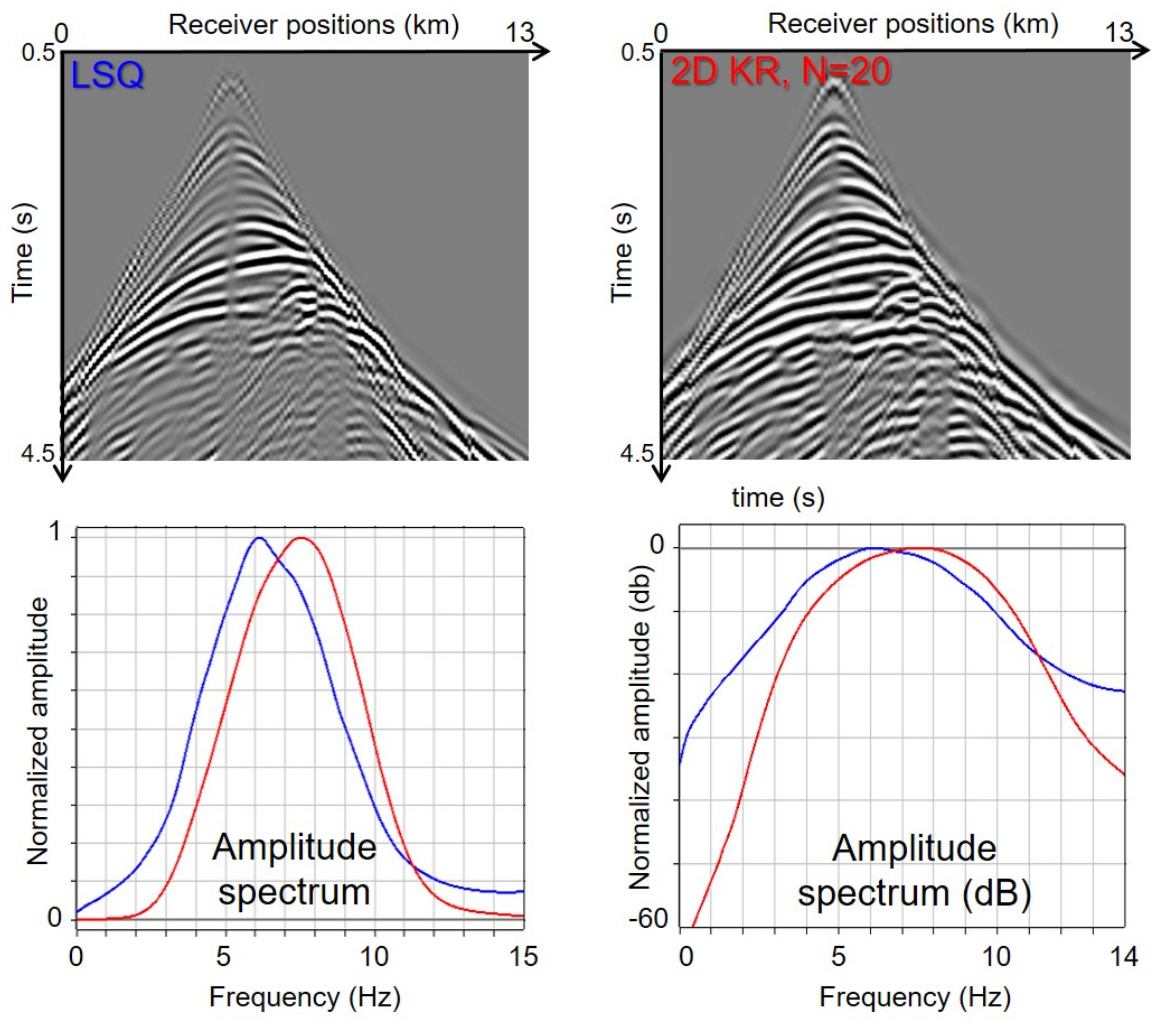

Figure 11. Same data as Fig. 10, but with the high-cut filter at $10 \mathrm{~Hz}$ (instead of the smooth high-cut filter above $4 \mathrm{~Hz}$ ). LSQ residual and 2D KR ajoint-source, together with their frequency spectra.
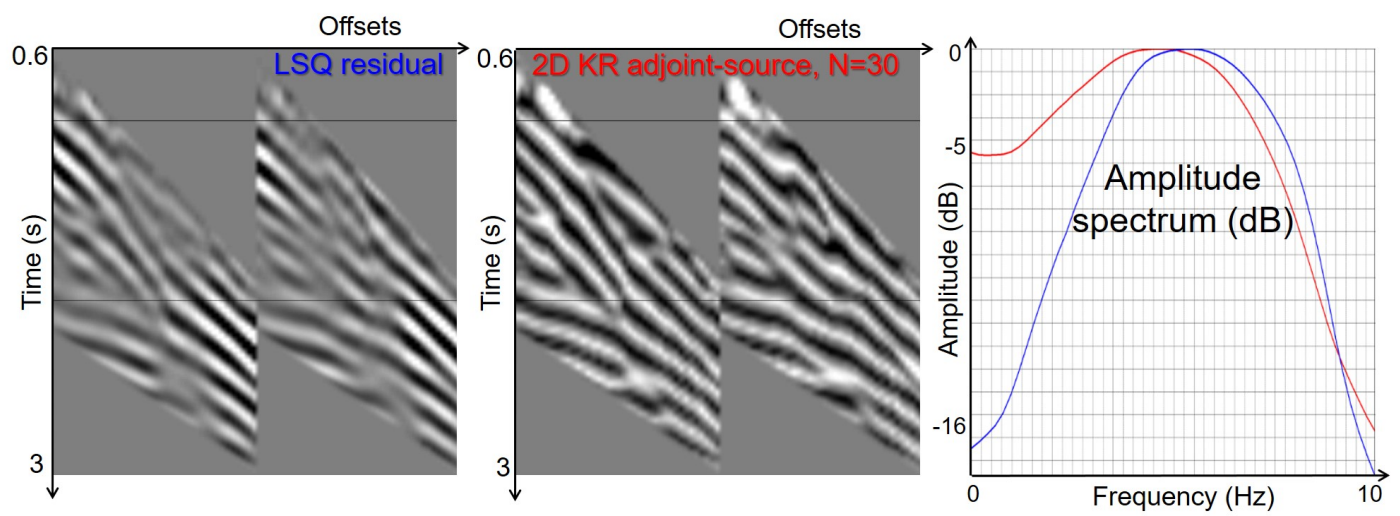

Figure 12. 3D field data set with a mute for a $6 \mathrm{~Hz}$ inversion. The two left figures represent the LSQ residual and the KR adjoint-source, muted as usual for the FWI. The right figure represents the corresponding amplitude spectra. Modified from Poncet et al. (2018) (see the article for more details).

\subsection{Thresholding and link with standard cost functions}

We now illustrate the effect of the thresholding constraint on common shot data. Choosing a good $\lambda$ value is important for the success of the scheme. Using a value that is too large would lead to instabilities (possible "singularities"), as explained in $§ 2.2$. Using a value that is too small would be equivalent to using a $L_{1}$ norm cost function. A good $\lambda$ value must let the 1Lipschitz constraint saturate at most positions in $X \backslash X_{\text {null }}^{(\Delta f)}$ and limit the additive constant (or null frequency) issue discussed in $\S 3.4 .3$. Within reasonable limits, slightly smaller $\lambda$ values would 
make the thresholding constraint saturate slightly more, producing a clipping of the adjoint-source and thus slightly reducing the sensitivity of the FWI to amplitudes.

Fig. 13 (bottom-right) illustrates the thresholding that occurs on Marmousi data when $\lambda$ is reduced; we can observe that the clipping remains slight (the amplitude dynamics are not strong with this data) and that larger $\lambda$ values may lead to a better amplitude equalization for a finite number of SDMM iterations (top-right).

We do not observe an additive constant or null frequency in our Marmousi KR adjoint-source results, contrary to Métivier et al. (2016b, 2016c). It is probable that this is due to our $\lambda$ tuning, that contribute to limit the additive constant effect as discussed in §3.4.3. A possible effect will be discussed further on the Marmousi FWI example in $\S 4.7$.

Note that the $N$ and $\lambda$ parameters allow us to consider KR as a hybrid cost function that mixes OT with the standard LSQ and $L_{1}$ cost functions, being able to pass continuously from one to the other.
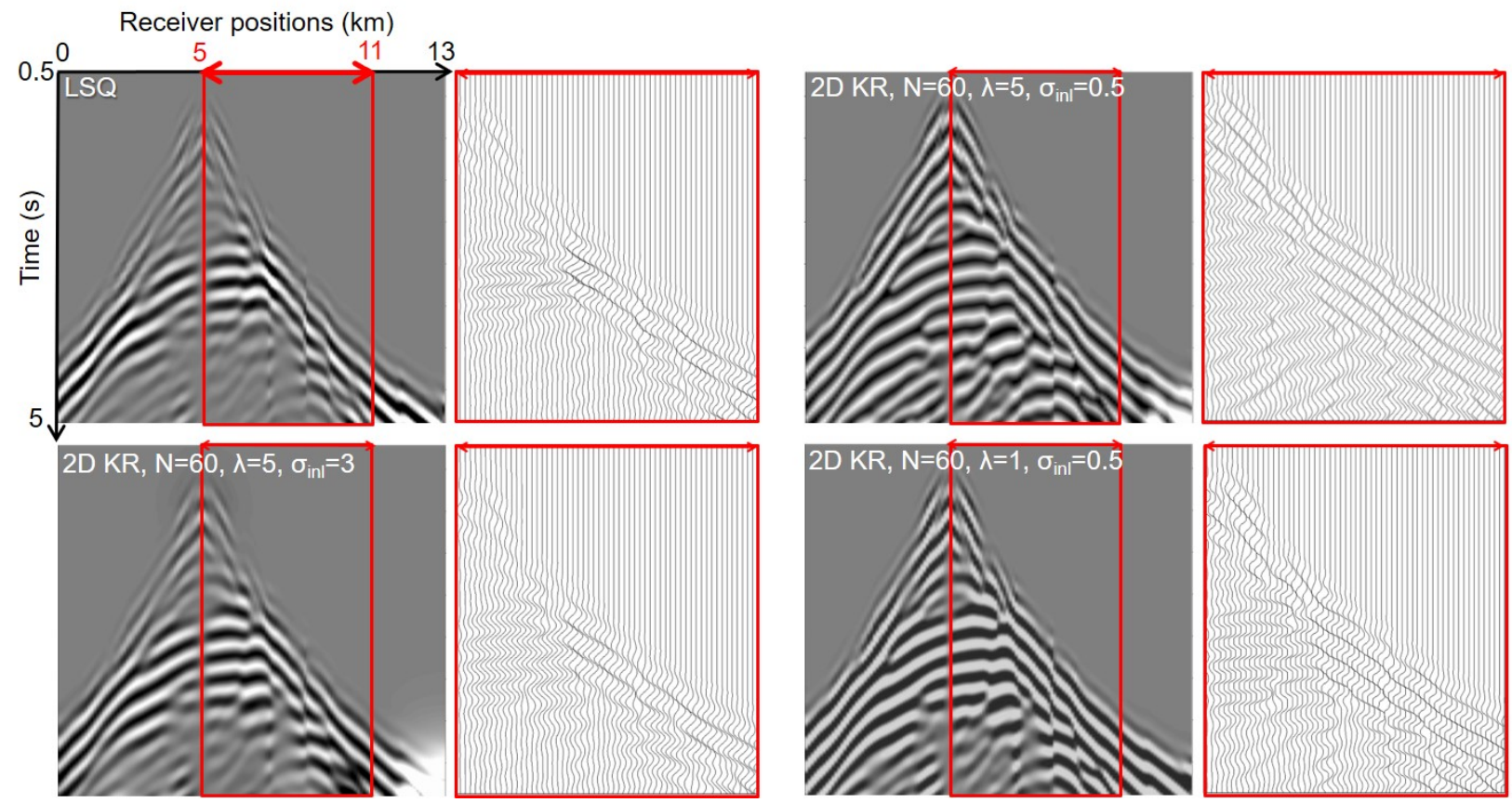

Figure 13. Same data as Fig. 10. LSQ residual and 2D KR adjoint-sources (effect of $\lambda$ and $\left.\sigma_{\text {inl }}\right)$ are shown.

\subsection{Improvement of the rate of convergence with one of the standard-deviation-like weights}

Let us come back to the chosen norm for the data coordinate space, i.e. eq. (13) with $p=1$. We have, in the $2 \mathrm{D}$ case

$$
\|x\|_{1}^{(X)}=\frac{1}{\sigma_{i n l}}\left(\left|x_{i n l}\right|+v\left|x_{t}\right|\right)=\frac{1}{\sigma_{t}}\left(\frac{1}{v}\left|x_{i n l}\right|+\left|x_{t}\right|\right) \quad \text { with } \quad v=\frac{\sigma_{i n l}}{\sigma_{t}},
$$

where $\sigma_{i n l}\left(\right.$ or $\sigma_{t}$ ) represents a standard-deviation-like weight in the inline (or time) direction, thus has the dimension of a distance (or a time), and $v$ represents an apparent velocity parameter.

It can be demonstrated that, at full convergence (i.e. a sufficiently large number $N$ of SDMM iterations), any choice for $\sigma_{i n l}$ (or $\sigma_{t}$ ) is equivalent when an optimal threshold ( $\lambda$ value) is considered, as the corresponding adjoint-sources would almost only differ by a global proportionality constant compensated by the line search. But, in practice for large scale applications, we can only run a small number of iterations $N$ (usually around 30-60). Hence, $\sigma_{\text {inl }}$ (or $\sigma_{t}$ ) can play a role, mostly related to a change of the rate of convergence. Fig. 13 illustrates the much slower convergence that occurs with a too large $\sigma_{i n l}$ value (the KR adjoint-source remains much closer to the LSQ adjoint-source for a given number of iterations $N=60$ ). This is thus not directly related to the $2 \mathrm{D}$ (or multiD) behavior in the data coordinate space. In $1 \mathrm{D}$, only the parameter $\sigma_{t}$ can play this role (the parameter $\sigma_{i n l}$ then does not exist). 


\subsection{Enhancement of the KR adjoint-source continuity with the apparent velocity (multidimensional feature)}

Now, let us define which parameter controls the multiD (here 2D) KR feature. For a fixed $\sigma_{i n l}$ (or $\sigma_{t}$ ), the velocity $v$ in eq. (34) plays a crucial role in the success of $2 \mathrm{D}$ KR FWI. It defines the average direction along which most correlations between inline traces events occur, thus must be parameterized to characterize the average moveout direction.

To better understand this point we note that, according to eq. (23), the 1-Lipschitz constraint corresponding to eq. (34) implies almost everywhere in $X$

$$
\left|\frac{\partial \varphi_{\max }(x)}{\partial x_{i n l}}\right| \leq \frac{1}{\sigma_{i n l}} \quad \text { and } \quad\left|\frac{\partial \varphi_{\max }(x)}{\partial x_{t}}\right| \leq \frac{v}{\sigma_{i n l}}
$$

Taking a very small $v$ value would constrain $\varphi_{\max }$ relative variations to be slower in the time direction than in the inline direction, possibly leading possible to vertical artifacts in the $2 \mathrm{D}$ KR adjoint-source; for sufficiently small $v$ and $\sigma_{i n l}$, the result becomes equivalent to the 1D KR adjointsource (up to an irrelevant global proportionality constant). Taking a large $v$ value would constrain $\varphi_{\max }$ relative variations to be slower in the inline direction than in the time direction, possibly leading to some horizontal artifacts. Taking an optimum $v$ value would tend to:

- Favor piecewise linearity of the KR adjoint-source in the time direction, the latter contributing the most to reduce the cycle-skipping issue.

- Within these bounds, favor the balance between the $\varphi_{\max }$ derivatives in the time and inline directions, i.e. favor the average moveout direction.

This is illustrated in Figs. 14-16.

Figs. 14 considers a simple synthetic 2D LSQ adjoint-source with events built from Ricker wavelets that are similar than these in Fig. 2b. However, theses events are here extended in a second (inline) dimension applying linear (first two events) and hyperbolic (last two events) moveouts. Then, each trace is shifted randomly in the time direction to degrades the continuity of the LSQ adjoint-source's events as visible in Fig. 14 (left). The 1D KR adjoint-source, while producing the KR texture for each trace, does not manage to recover the continuity in the moveout direction. In contrast, the 2D KR adjoint-source corresponding to an optimum $v$ manages to also recover a much better continuity, producing a denoising effect that enhances even more the kinematic information present in the data, which is beneficial to FWI. However, without a suitable choice for $v$, the 2D KR adjoint-source can exhibit striping artefacts to the point of becoming unusable (Fig. 14, right).

Fig. 15 illustrates the avantage of $2 \mathrm{D} \mathrm{KR}$ on a field marine data. This data is polluted by a noise that is different from one trace to the other. Note that a mute is applied to the data, common in industrial FWI to help the inversion concentrate on the most relevant data, i.e. the diving waves. Fig. 15 (top) shows that the noise in the data degrades the continuity of LSQ adjointsource and that the 1D KR adjoint-source does not recover any better continuity. In contrast, the $2 \mathrm{D} \mathrm{KR}$ adjoint-source with an optimum $v$ is strongly denoised, with an increased continuity in the moveout direction and better amplitudes balancing. This may be useful to start FWI at even lower frequency and to relax sensitivity to amplitudes. Fig. 15 (bottom) shows how $v$ affects the 2D KR adjoint-source and how a good choice enhances the continuity of events.

Finally, Fig. 16 illustrates on a synthetic Marmousi adjoint-source that 2D KR with a good $v$ choice allows to produce an adjoint-source with an enhanced continuity of events in the moveout direction, despite the absence of noise in the data. The effect is then more subtle but nevertheless allows to improve the Marmousi inverted model as further in this section.

We thus observe that accounting for correlations between group of traces in a well sampled or non aliased spatial direction allows to exploit more information than only the time related the events, like here information related to events continuity. 

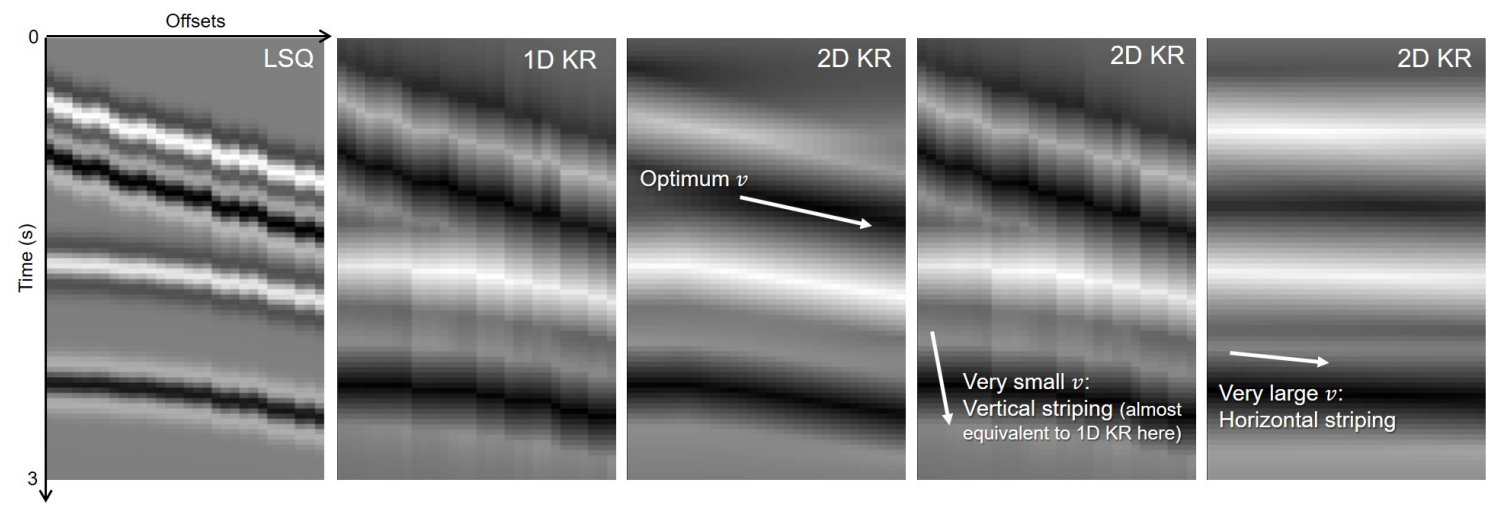

Figure 14. Comparison of adjoint-sources for a simple example, where $f_{\text {obs }}$ contains events built from Ricker wavelets (peak frequency at $6 \mathrm{~Hz}$ ) and $f$ contains the same events but shifted. The setting is similar than in Fig. $2 \mathrm{~b}$ but the events are extended in a second (inline) dimension applying linear and hyperbolic moveouts, and then the traces are randomly shifted in time to degrade the continuity. LSQ residual, 1D KR adjoint-source, and 2D KR adjoint-sources (effect of $v$ ) are shown.

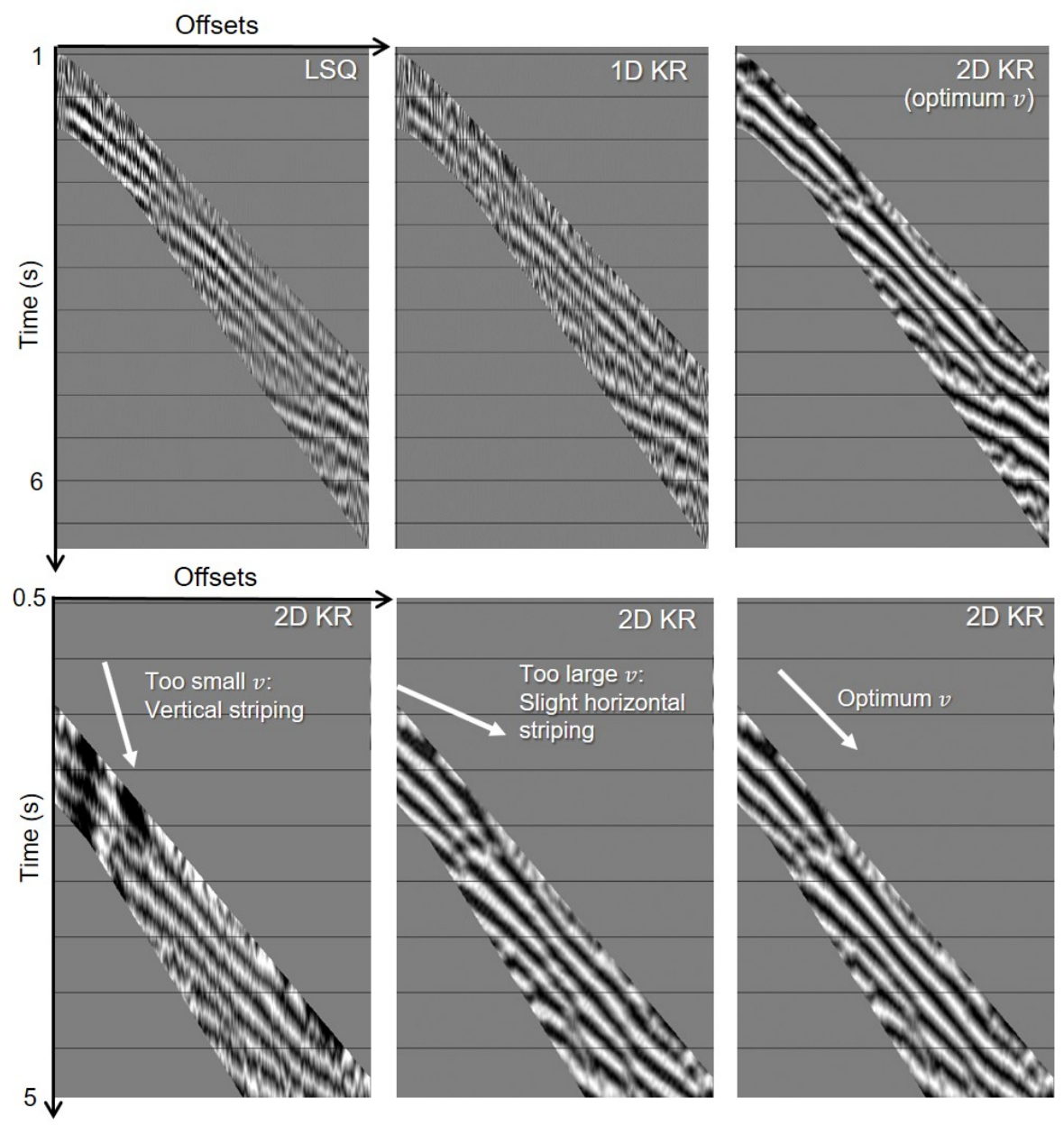

Figure 15. Marine field data at $4 \mathrm{~Hz}$. LSQ residual, 1D KR adjoint-source, and 2D KR adjoint-sources (effect of $v$ ) are shown. 

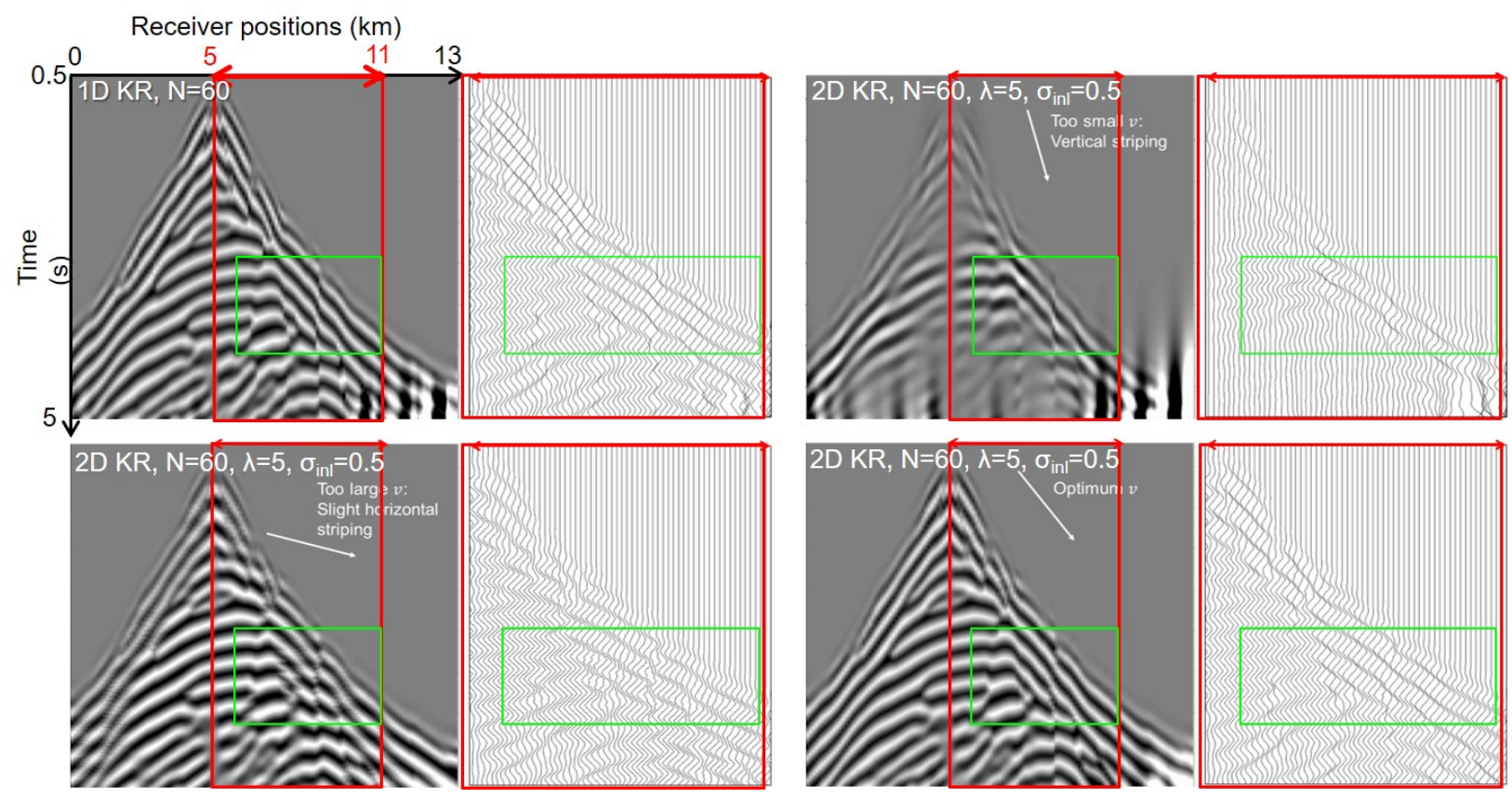

Figure 16. Same data as Fig. 10. 1D KR adjoint-source and 2D KR adjoint-sources (effect of $v)$ are shown.

\subsection{FWI adaptations and interest of $K R$ for the inversion}

Up to now, the analysis has remained at the adjoint-source level, which has allowed us to understand many features of the use of the KR norm within FWI. In the rest of this article, we illustrate how these features translate into a better velocity update. A SDMM number of iterations $N=30$ is considered in all the following results.

Once the gradient in the model space has been computed using the adjoint-state method described in $\S 2.1$, it is common to apply to it a diagonal preconditioning followed by a L-BFGS preconditioning and a line search (Plessix, 2006; Virieux and Operto, 2009). The cost function choice directly affects the adjoint-source only, but it indirectly affects the FWI through the preconditioning and the line search. For instance, the derivation of the classical FWI gradient diagonal preconditioning of Warner et al. (2013) is only valid for the LSQ cost function. However, we have not found any pratical downsides of using it for KR FWI. Similarly, a single line-search iteration is valid only for the LSQ cost function, with a Hessian equal to the identity; but the line-search can be iterated when using non LSQ cost functions.

We firstly consider the Marmousi 2 data set (Martin et al., 2006). Like above, a Ricker wavelet with a peak frequency at $6 \mathrm{~Hz}$ and all frequencies below $3 \mathrm{~Hz}$ muted is used (the adjoint-source examples in Fig. 11 are related to this case). The constant-density acoustic approximation has been used for the modelling and only the velocity is inverted by the FWI. 20 FWI iterations were performed directly at up to $10 \mathrm{~Hz}$, keeping all the data (first break, reflections, multiples...) and starting with a smooth initial velocity model where LSQ is cycle-skipped (obtained by Gaussian filtering of the true model), see Fig. 17. The model estimated by $1 \mathrm{D} \mathrm{KR}$ matches the true model better than LSQ, especially in the highlighted zones, mitigating cycle-skipping and producing more continuity along structures, see Fig. 17. The texture of the KR adjoint-source (including the enhanced low frequency content) helps overcome cycle-skipping. (The strength of this effect may depend on the extent of the low frequencies present in the forward propagated source wavelet; sufficient coupling occured in our experiments.)

Note that our Marmousi 1D KR FWI result in Fig. 17 looks improved compared to Fig. 27d in Métivier et al. (2016b). This may be due to the additive constant or null frequency observed in the Marmousi adjoint-sources of Métivier et al. (2016b) (see Fig. 26b of the latter article for a $2 \mathrm{D} \mathrm{KR}$ adjoint-source). In practice, this could produce a low frequency smearing that may somewhat overlap the low frequencies present in the forward propagated source wavelet; then, if 
the low frequency smearing is different from one trace to the other, and as 1D KR treats each trace independently, this may degrade the 1D KR FWI update. As discussed in $\S 4.4$, we do not observe such an additive constant in our KR adjoint-source results, that may explain our improved Marmousi 2 model update. However, the conclusion remains the same: the 2D KR FWI result is better than the 1D KR FWI result, producing a better structural coherency. The 2D KR adjointsource continuity enhancement (achieved with the $v$ parameter) maps into a FWI model update structural coherency enhancement. This remains true even if there is not a lot of noise in the data, i.e. when the adjoint-source improvement brought by $2 \mathrm{D} \mathrm{KR}$ compared to $1 \mathrm{D} \mathrm{KR}$ is more subtle than in the case of Fig. 15 for instance.
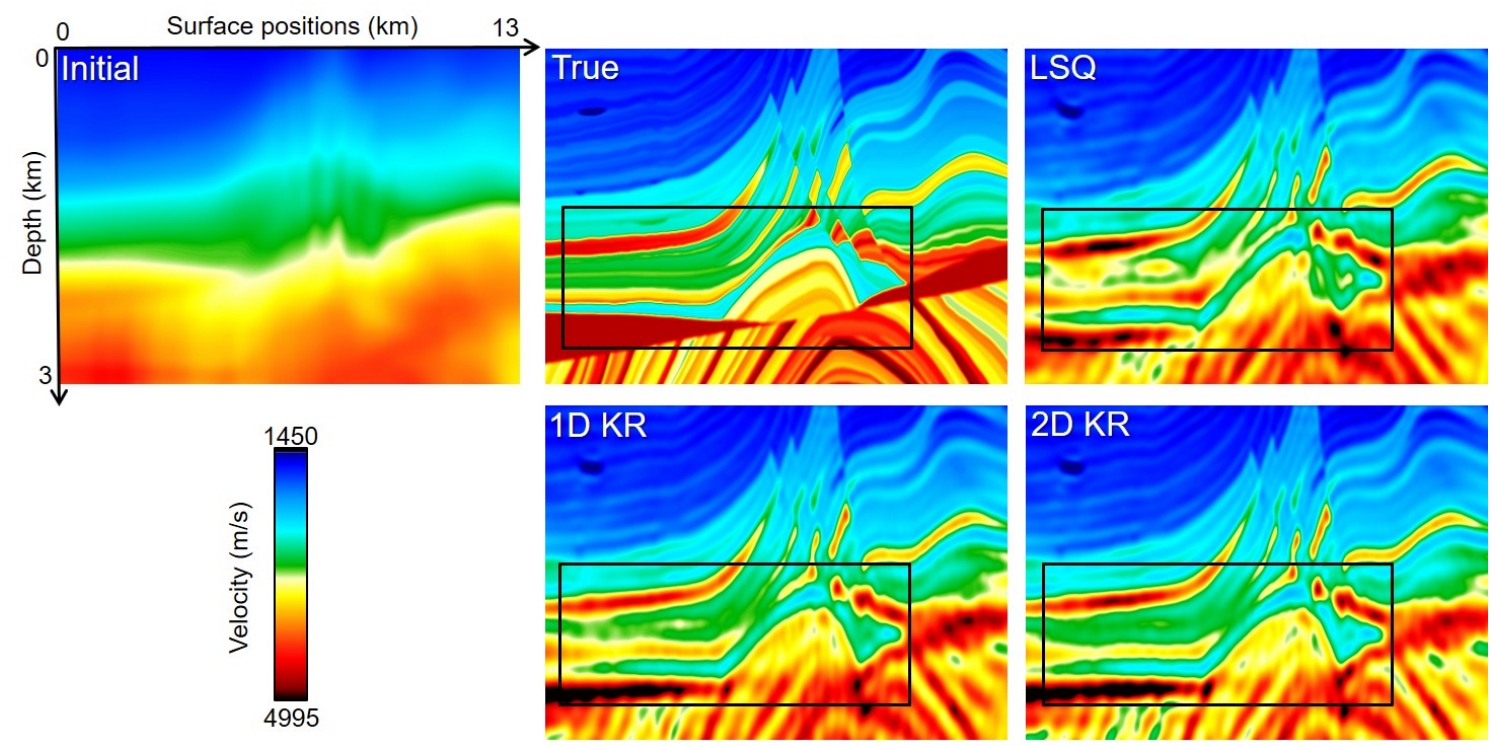

Figure 17. Marmousi 2 model (Martin et al., 2006). FWI inversion directly at $10 \mathrm{~Hz}$ (adjointsources in Fig. 11 are related to this case).

\section{Application to field data}

Successful applications of KR FWI on large scale field data have been published in (Poncet et al., 2018; Messud and Sedova, 2019; Sedova et al., 2019; Hermant et al., 2019; Carotti et al., 2020; Hermant et al., 2020). In this section, we gather few illustrations from Messud and Sedova (2019) and Carotti et al. (2020). For further details or more illustrations, we invite the reader to refer to the aforementioned articles.

\subsection{Land data}

The first example refers to an Oman land broadband full azimuth data set consisting of three separate acquisitions that have been merged, with challenges associated with irregular offset distributions among the merged surveys. Fig. 18 shows various FWI results obtained at $9 \mathrm{~Hz}$. LSQ FWI leads to poor structural consistency and poor fit to the sonic log at the location of the well. 2D KR FWI gives a better fit to the sonic log and a better consistency with the geology compared to LSQ FWI. This can be related to a reduced sensitivity to cycle-skipping and to the ability of 2D KR to enhance the continuity along structures. Fig. 19 compares 2D KR FWI to 1D KR FWI on the same survey. As a careful preconditioning of the data has been performed before FWI (to enhance events coherency, signal to noise ratio and thus convergence of FWI), following the workflow proposed by Sedova et al. (2019), the 1D KR adjoint-source looks quite similar to the 2D KR adjoint-source (not shown here). 1D KR FWI is capable of resolving the cycle-skipping present in LSQ FWI. However, 2D KR FWI leads to a better model update than 1D KR FWI, with better structural consistency and well matching. We underline that the level of improvement 
of 2D KR FWI over 1D KR FWI needs to be considered in the frame of the preconditioning steps applied to the data.

The second example refers to a 3D land broadband data set with full-azimuth and offsets of up to $13 \mathrm{~km}$, again acquired in Oman. This data set was processed to enhance diving and post-critical waves. The FWI has been run with the frequency increasing from $2 \mathrm{~Hz}$ to $16 \mathrm{~Hz}$, following the workflow proposed by Sedova et al. (2019). Fig. 20 compares LSQ FWI to 2D KR FWI. The yellow oval in Fig. 20 highlights the improved delineation of the velocity contrast achieved by 2D KR FWI, and the unexpected velocity increase obtained with LSQ FWI that is corrected by 2D KR FWI. This improved velocity provides imaging uplifts of the deep reflectors observable in Fig. 20b (right). Moreover, the focusing of the major fault, a difficult challenge for the area, is enhanced, as highlighted by the yellow arrow in Fig. 20b (right).

\subsection{Marine data}

The third example refers to a North Sea marine data set. Fig. 21 shows results obtained with a $7 \mathrm{~Hz}$ FWI inversion. LSQ FWI is cycle-skipped, as indicated by the "red spots" in the observed data overlaid on top of the modelled data (highlighted by green arrows). These red spots are due to events that suddently jump from one "cycle" to another in the modelled data, resulting in a lack of structural consistency and continuity in the inverted velocity model. Fig. 21 shows how 2D KR FWI solves for these issues and inverts for an improved velocity model (especially in the areas highlighted by green arrows).

The last example refers to a Barents Sea marine data set, that is challenging because of gas accumulations of varying size and depth location. Fig. 22 illustrates the stability of $2 \mathrm{D}$ KR FWI over LSQ FWI, the latter being cycle-skipped. Even with a poor initial model, 2D KR FWI converges to a more structurally consistent model update, while mitigating cycle-skipping and matching better the observed data. 

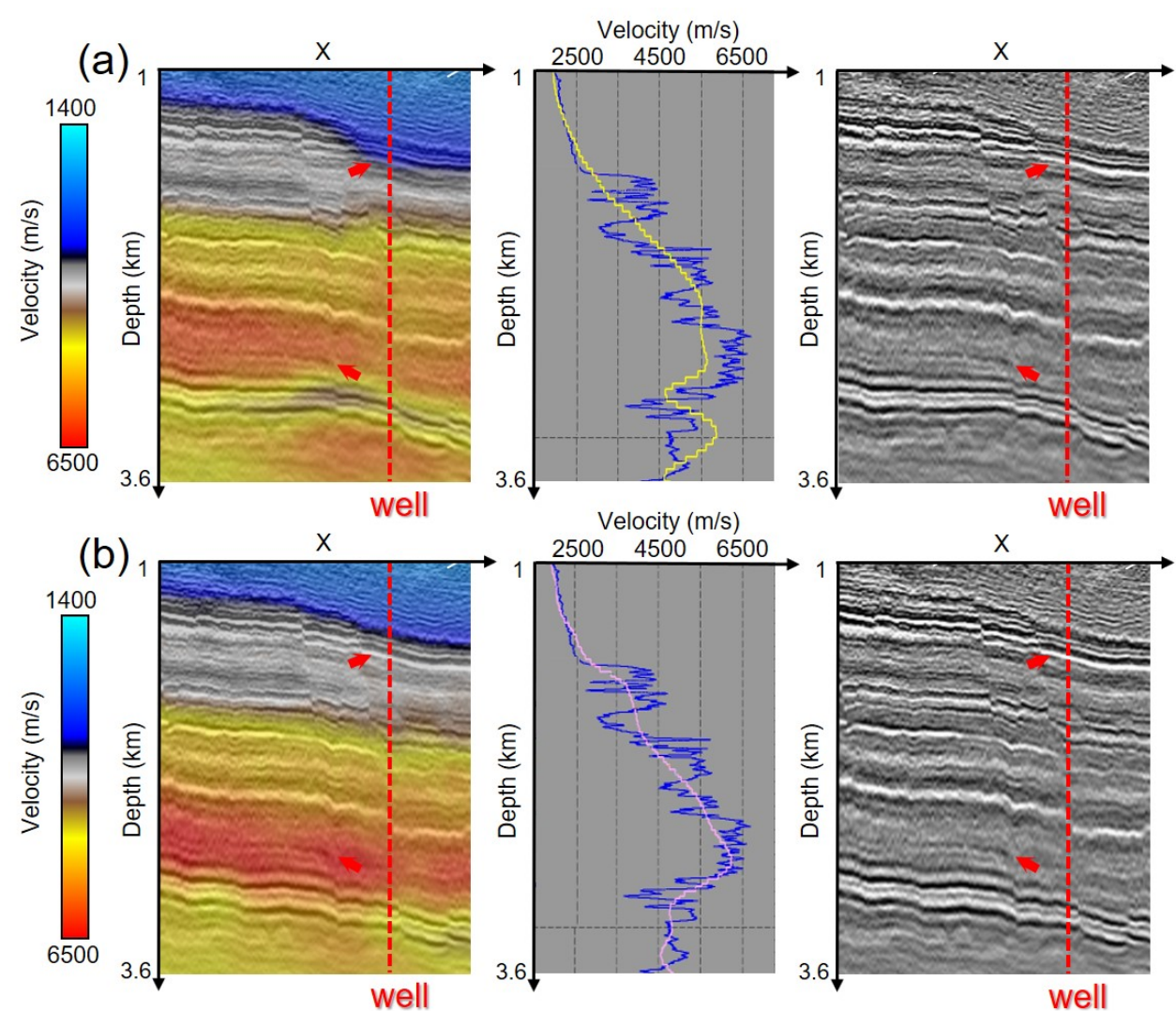

Figure 18. FWI results at $9 \mathrm{~Hz}$ on Oman land data. (a) LSQ FWI result and (b) 2D KR FWI result (same input data, initial model, mute and number of iterations). Three images are presented for each case. Left: velocity model superimposed on the corresponding Kirchhoff depth migrated stack. Middle: well measurement (blue) and inverted model trace at the position schematized by the dashed red line. Right: Kirchhoff depth migrated stack. Modified from Messud and Sedova (2019) (see the article for more details).
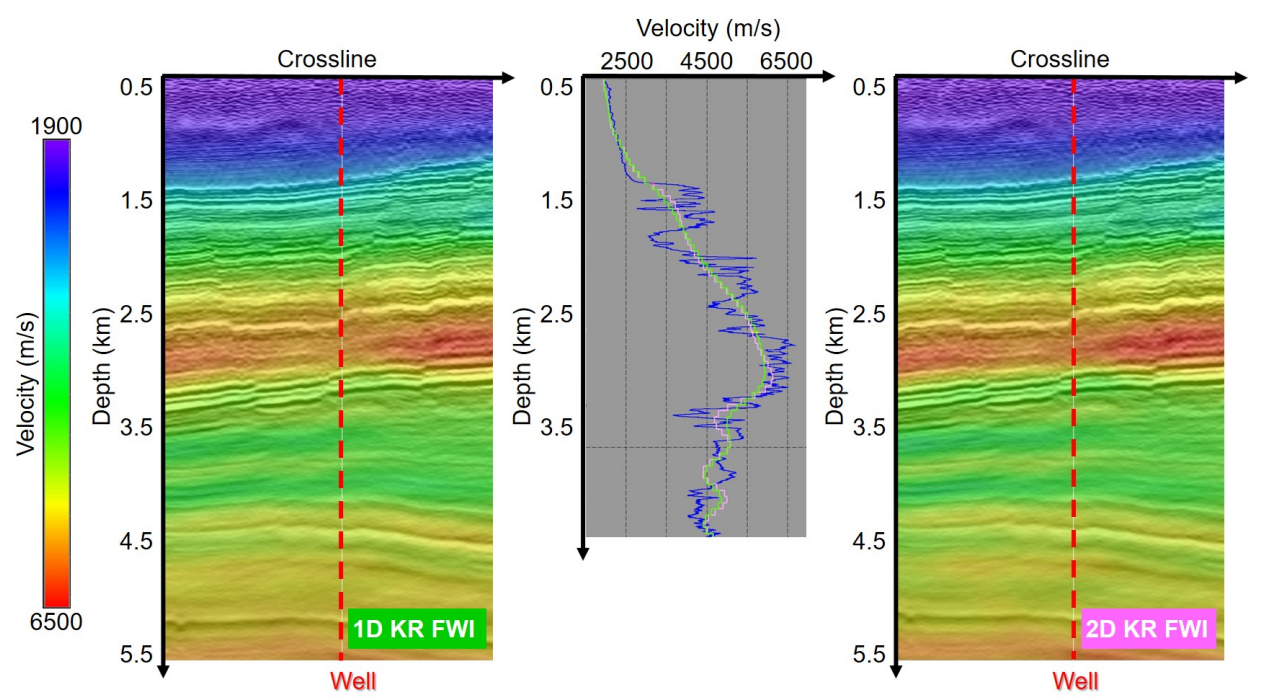

Figure 19. FWI results at $9 \mathrm{~Hz}$ on Oman land data. Left, right: models inverted at $9 \mathrm{~Hz}$ superimposed on the migrated stacks (same input data, initial model, mute and number of iterations). Middle: well measurement (blue) and inverted model traces (green is 1D KR and magenta is 2D KR) at the position schematized by the dashed red line. Modified from Messud and Sedova (2019) (see the article for more details). 

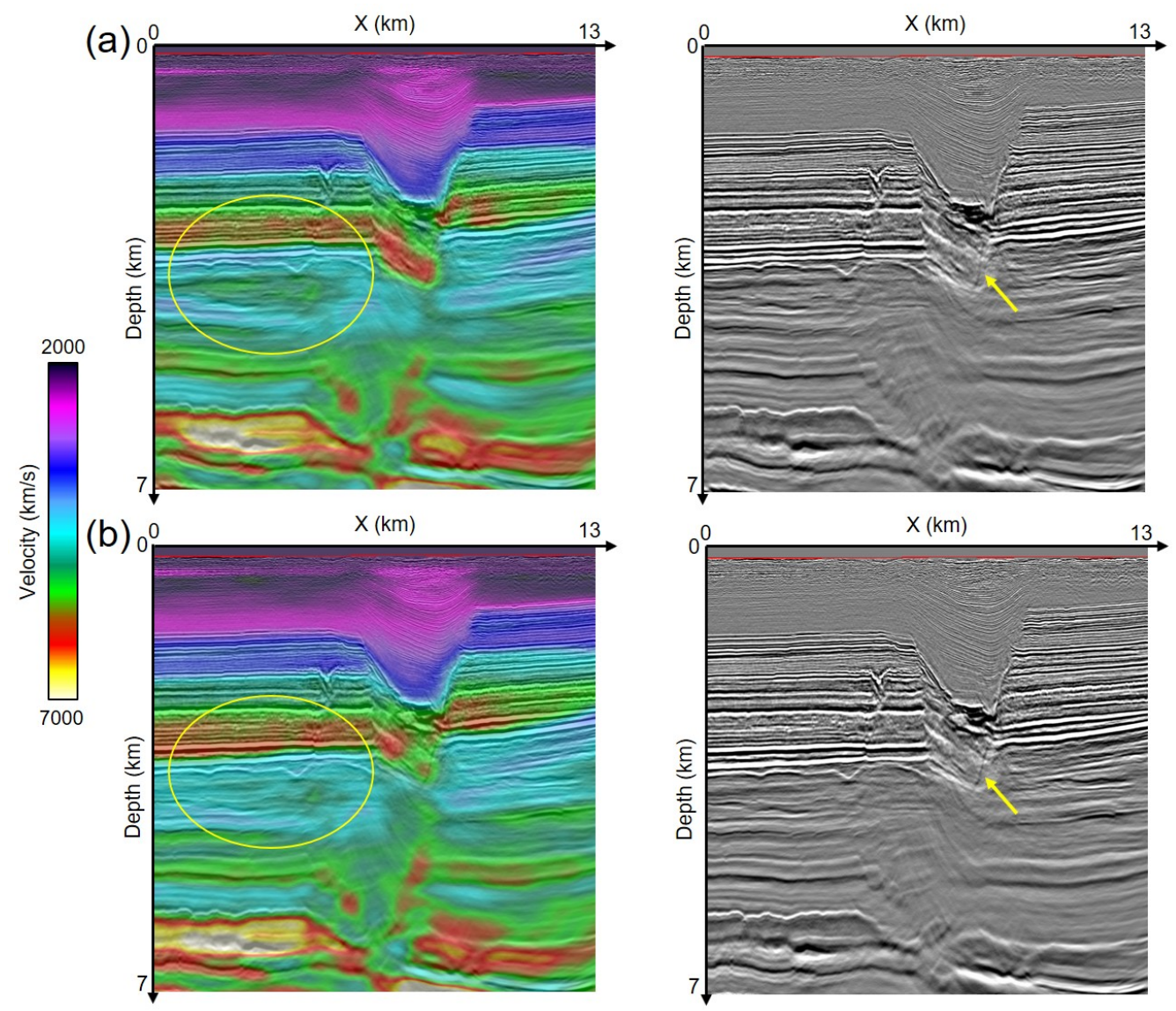

Figure 20. FWI results at $16 \mathrm{~Hz}$ on North of Oman land data. (a) LSQ FWI result and (b) 2D KR FWI result (same input data, initial model, mute and number of iterations). Two images are presented for each case. Left: velocity model superimposed on corresponding Kirchhoff depth migrated stack (the yellow ovals highlight the improved delineation of the velocity contrast and the correction of the velocity increase achieved by 2D KR FWI). Right: Kirchhoff depth migrated stack (the yellow arrows highlight the improved focusing of the major fault achieved by $2 \mathrm{D}$ KR FWI). Modified from Carotti et al. (2020) (see the article for more details). 

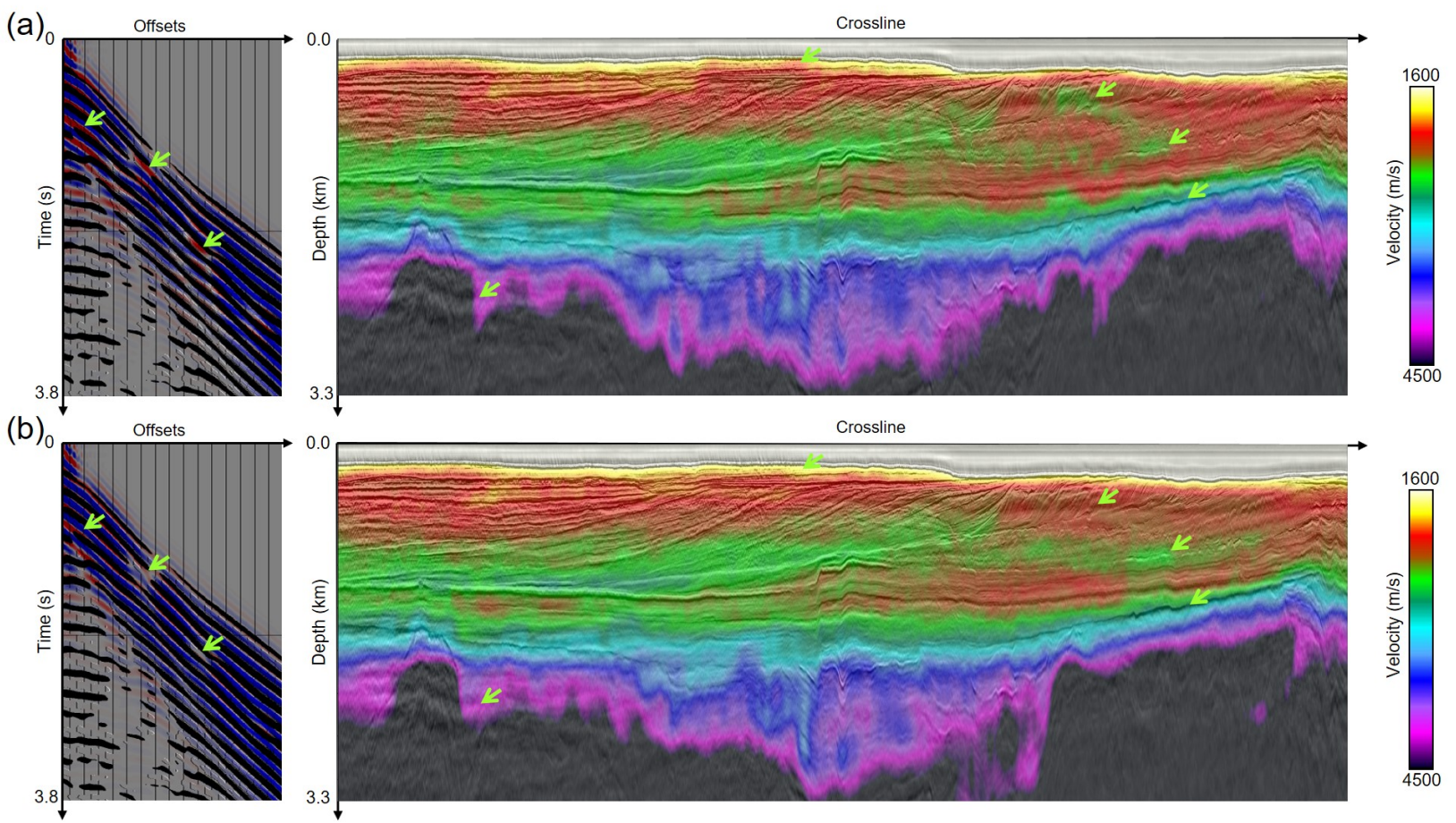

Figure 21. North sea data. (a) LSQ FWI result and (b) 2D KR FWI result (same input data, initial model, mute and number of iterations). Two images are presented for each case. Left: observed data (in black-grey-white) superimposed to modelled data (in red-blue) (the green arrows highlight where the modelled data suddently jumps from one "cycle" to another with LSQ). Right: FWI updated models at $7 \mathrm{~Hz}$ (the green arrows highlight some areas where $2 \mathrm{D} \mathrm{KR}$ FWI gives an improved and more structurally consistent velocity model). Modified from Messud and Sedova (2019) (see the article for more details). 

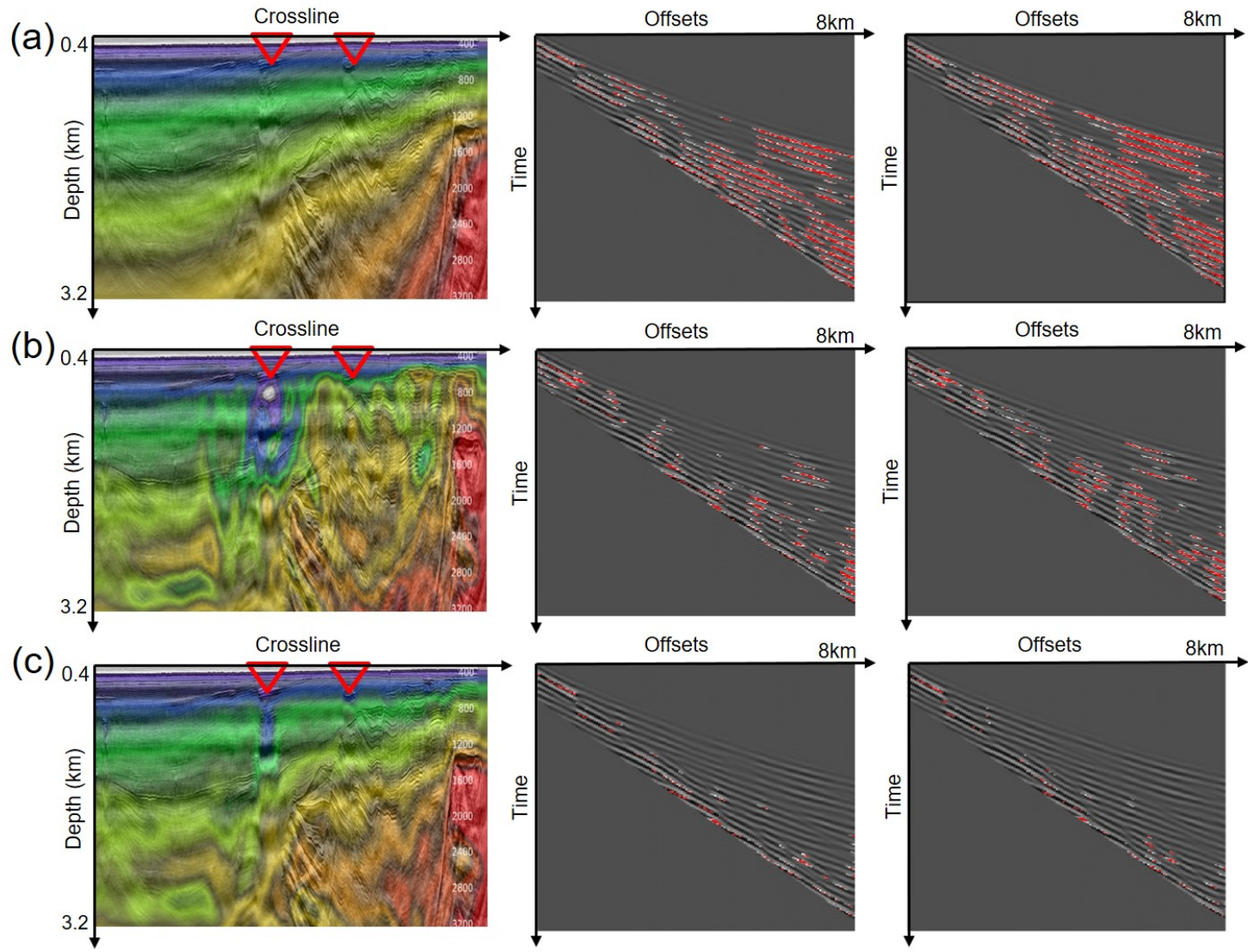

Figure 22. Barents Sea data. (a) Initial model for FWI, (b) LSQ FWI result and (c) 2D KR FWI result (same input data, initial model, mute and number of iterations). Three images are presented for each case. Left: velocity model and migrated stack. Middle and right: normalized absolute values of the difference between observed and modelled data (red means large values thus poor matching), at the two positions highlighted by the red triangles. Modified from Carotti et al. (2020) (see the article for more details).

\section{Perspectives}

We end up by providing some perspectives and come-back to adjoint-source considerations. Examining previous KR adjoint-sources, e.g. Figs. 3-15, we notice that:

- The kinematics present in the adjoint-source is enhanced by the KR texture (enhanced low frequencies, more balanced amplitudes and events continuity), explaining why the KR norm is more robust to cycle-skipping and amplitudes.

- But the kinematics present in the adjoint-source is not modified, unlike other methods that help to reduce the cycle-skipping issue by shifting events in the data and using the corresponding kinematically-modified residual. See e.g. the method of Baek et al. (2014) and Wang et al. (2016) that we call "registration-guided" FWI in the following (sometimes also called "dynamic warping" FWI), or the method of Métivier et al. $(2018,2019)$ called "graph-space" (GS) OT FWI.

Ultimately, we would like to bring both features into a common framework to combine their advantages (texture change together with kinematics change) and become even more robust to cycle-skipping. This is possible by introducing the concept of "kinematic transformation" (KT) into the KR norm.

We call KT any transformation that makes the kinematics of the events present in the observed data $f_{\text {obs }}$ closer to the kinematics of another (relatively close) data $f$, usually the modelled data. We consider in this section the example of a KT that works in the timedirection only, with generalization being straightforward. $f_{o b s}\left(x_{x l}, x_{i n l}, x_{t}\right)$ is transformed into 
$f_{\text {obs }}\left(x_{x l}, x_{i n l}, \sigma[f]\left(x_{t}\right)\right)=f_{\text {obs }}^{(\sigma)}\left(x_{x l}, x_{i n l}, x_{t}\right)$ to become kinematically closer to a data $f\left(x_{x l}, x_{i n l}, x_{t}\right)$ from a certain similarity measure point of view. $\sigma[f]:] 0, T] \rightarrow] 0, T]$ represents the KT, a functional of the data whose kinematics should be (partially) matched. The registration-guide (Baek et al., 2014; Wang et al., 2016; Hale, 2013) and the permutation resulting from GS (Métivier et al., 2019) are examples of KT. Used within FWI, the goal is to change the kinematics of the observed data to make it closer to the modelled data at each FWI iteration, to improve robustness to cycle-skipping.

Our goal here is not to detail the differences between KT but to give a framework, so we remain general. To embed a KT into KR, we can simply consider instead of eq. (14):

$$
\tilde{W}_{d}\left(f, f_{o b s}^{(\sigma)}\right)=\max _{\varphi \in \operatorname{BLip}\left(\|\cdot\|_{1}^{(X)}, 1, \lambda\right)} \int_{X} \varphi(x)\left(f\left(x_{x l}, x_{i n l}, x_{t}\right)-f_{o b s}\left(x_{x l}, x_{i n l}, \sigma[f]\left(x_{t}\right)\right)\right) d \mu(x),
$$

where $\sigma[f]$ is defined by any KT method. Applying the considerations of $\S 3.1$ to eq. (36), we can consider the following expression as the adjoint-source

If

$$
\frac{\partial \tilde{W}_{d}\left(f, f_{o b s}^{(\sigma)}\right)}{\partial f(x)}=\varphi_{\max }[f](x)-\int_{X} \varphi_{\max }[f]\left(x^{\prime}\right) \frac{\partial f_{o b s}\left(x_{x l}^{\prime}, x_{i n l}^{\prime}, \sigma[f]\left(x_{t}^{\prime}\right)\right)}{\partial f(x)} d \mu\left(x^{\prime}\right)
$$

$$
\exists \gamma>0, \forall h \in L^{1}(X) \text { such as }\|h\|_{1}<\gamma: \sigma[f+h](x)=\sigma[f](x),
$$

i.e. if $\sigma[f]$ is insensitive to infinitesimal perturbations of $f$, the second term in eq. (37) can be neglected and the adjoint-source can keep the simple form $\varphi_{\max }[f]$ used in KR FWI without KT (the adjoint-source form then would remain the same, but not the adjoint-source itself as the KT would affect the $\varphi_{\max }$ values through eq. (36)). So, do some existing KT fulfill eq. (38)?

- It seems not strictly the case for the registration-guide (Baek et al., 2014; Wang et al., 2016), even if in practice the scheme neglects $\partial f_{o b s}\left(x_{x l}^{\prime}, x_{i n l}^{\prime}, \sigma[f]\left(x_{t}^{\prime}\right)\right) / \partial f(x)$ "by hand", arguing it represents a second order term, non-crucial because of the non-linearity of the FWI.

- But it is strictly the case for GS as demonstrated formally in Métivier et al. (2019) (in a discrete $X$ space case as GS only deals with discrete data coordinate spaces). This is a result in favor of the use of GS as a KT within KR FWI, at least mathematically. Physically, the pertinence of GS OT FWI compared to registration-guided FWI still needs to studied.

To conclude with an illustration, Fig. 23 (left) shows how the registration-guided KT can affect the LSQ residual. We notice that the texture remains quite the same but the kinematics of some events in the residual change, especially in the diving wave area. On the contrary, the texture of the 2D KR adjoint-source is quite different from the LSQ residual, but the kinematics of the events does not change. Fig. 23 (right) shows the KR adjoint-source with a KT embedded; we can observe the KR texture together together with some events kinematics change. Embedding a KT can thus naturally "augment" the KR scheme, possibly for even more robustness to cycle-skipping. Comparing various possible KTs goes beyond the scope of this article. We mention this point as a perspective, that has started to be explored in Kpadonou et al. (2021).

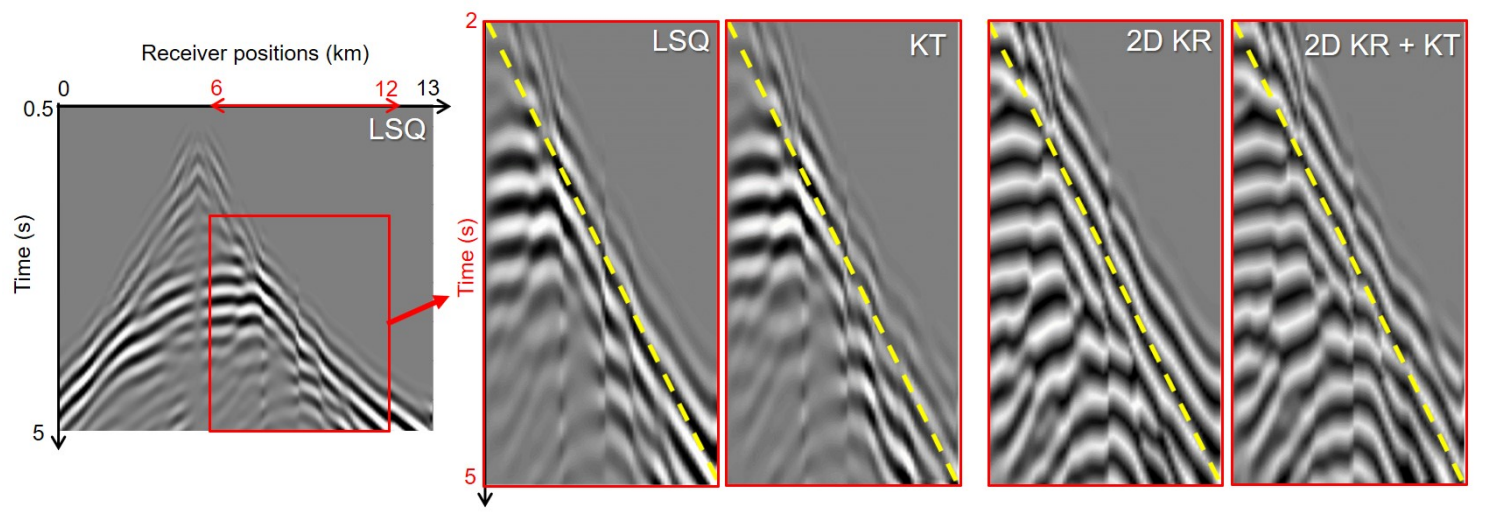

Figure 23. Same data as Fig. 10, zoom. Various adjoint-sources compared: LSQ, registrationguided KT, 2D KR and 2D KR-embedded registration-guided KT. The yellow dashed line helps comparing the kinematic change brought by the KT in the diving waves area. 


\section{Conclusion}

The use of the KR norm in FWI is now well established and has proven its benefits for practical large-scale applications. Thanks to a significantly wider global minimum valley, several field data case studies have shown that KR FWI clearly outperformed LSQ FWI in overcoming moderate cycle-skipping. In this article, we have developed an analysis to better understand the benefits of the KR norm for FWI, mainly focusing on the adjoint-source. We have addressed both theoretical aspects, fundamentally justifying the KR adjoint-source expression and qualifying its "texture", and practical points that are critical for an efficient use within FWI. The KR adjoint-source can be conceptualized as the result of smart processing applied to the LSQ residual, that enhances the low frequency content and reduces the dynamics of amplitudes. When correctly tuned, the 2D (or multiD) KR adjoint-source appears to also enhance the lateral continuity of events (in a well sampled or non aliased spatial direction) within a common shot gather. All these components, reinforcing the kinematic content in the adjoint-source, are beneficial to a successful FWI implementation.

If the use of the KR norm within FWI significantly widens the global minimum valley compared to the use of LSQ, it does not fully solve the cycle-skipping issue or the challenge of FWI with reflected waves only. Further investigations in these directions are ongoing (Métivier et al., 2019; Sun and Al Khalifah, 2019; Tang et al., 2020; Kpadonou et al., 2021).

\section{Acknowledgments}

We are grateful to CGG for granting permission to publish this work and to CGG Multi-Client, TGS, Occidental Petroleum, PDO, the Ministry of Oil and Gas of the Sultanate of Oman and INEOS for providing the data and permission to present the field data results. We are grateful to Klaas Koster for help and support and to Ludovic Métivier for enlightening discussions. We are indebted to Félix Kpadonou, Anna Sedova, Nabil Masmoudi, Adel Khalil and Roger Taylor for collaboration and enlightening discussions. We are indebted to Thibault Lesieur and Thibaut Allemand for collaboration and enlightening advice regarding Appendix A. 


\section{Appendix A. Dual representation of the Lipschitz constraint (proof)}

We consider $d \rightarrow\|\|_{p}^{(X)}$ in the Lipschitz constraint, eq. (11), and $(x, y) \in X \times X$. The Lipschitz semi-norm is defined by

$$
\|\varphi\|_{L_{i p_{p}}}=\max _{x \neq y} \frac{|\varphi(x)-\varphi(y)|}{\|x-y\|_{p}^{(X)}} .
$$

We obtain a norm identifying functions $\varphi$ defined up to an additive constant.

Taking $x=y+z$ with $z \in X$ and choosing a $\varphi(y)$ that is differentiable, the Lipschitz norm can be rewritten $(p \geq 1)$

$$
\begin{aligned}
\|\varphi\|_{\text {Lipp }} & =\max _{y} \max _{z \neq 0} \frac{|\varphi(y+z)-\varphi(y)|}{\|z\| \|_{p}^{(X)}}=\max _{y} \max _{z \neq 0} \frac{\left|\frac{\partial \varphi(y)}{\partial y} \cdot z\right|}{\|z\|_{p}^{(X)}}=\max _{y} \max _{\|z\|_{p}^{(X)} \leq 1}\left|\frac{\partial \varphi(y)}{\partial y} . z\right| \\
& =\max _{y}\left\|\frac{\partial \varphi(y)}{\partial y}\right\|_{q}^{(X)} \quad \text { with } \quad 1 / p+1 / q=1 .
\end{aligned}
$$

The last equality is obtained by definition of the dual norm (Rudin, 1991; Brezis, 2020). The Lipschitz norm can thus be "represented" by a $L_{q}$ norm $\|.\|_{q}^{(X)}$ on $\partial \varphi(y) / \partial y$.

Let us consider standard-deviation-like weights that satisfy $\infty>1 / \sigma>\epsilon$ where $\epsilon>0$. When the inverses of the $\sigma$ are introduced in $\|.\|_{p}^{(X)}$, i.e.

$$
\|x\|_{p}^{(X)}=\left(\frac{\left|x_{x l}\right|^{p}}{\sigma_{x l}^{p}}+\frac{\left|x_{i n l}\right|^{p}}{\sigma_{i n l}^{p}}+\frac{\left|x_{t}\right|^{p}}{\sigma_{t}^{p}}\right)^{1 / p},
$$

eq. (A.2) imposes that the non-inverted $\sigma$ appear in $\|\cdot\|_{q}^{(X)}$, i.e.

$$
\left\|\frac{\partial \varphi(x)}{\partial x}\right\|_{q}^{(X)}=\left(\sigma_{x l}^{q}\left|\frac{\partial \varphi(x)}{\partial x_{x l}}\right|^{q}+\sigma_{i n l}^{q}\left|\frac{\partial \varphi(x)}{\partial x_{i n l}}\right|^{q}+\sigma_{t}^{q}\left|\frac{\partial \varphi(x)}{\partial x_{t}}\right|^{q}\right)^{1 / q} \quad \text { with } 1 / p+1 / q=1 .
$$

Note that the second equality in eq. (A.2) is valid when the Riesz representation theorem can be invoked in $X^{*}\left(\left(\right.\right.$ Brezis, 2020) chapter IV), where $X^{*}=\mathcal{L}(X, \mathbb{R})$ denotes the (topological) dual of $X$. This is the case for any norm in finite dimensional spaces like $X$ that is 3 dimensional here. (For completeness, we remind that this is not the case in infinite dimensional space (Rudin, 1991; Brezis, 2020)).

As the 1-Lipschitz constraint implies strong continuity, i.e. differentiability almost everywhere, the derivative $\partial \varphi(x) / \partial x$ is defined almost everywhere. Constraining the derivative almost everywhere is sufficient for the Lagrange multipliers theoretical considerations of Appendix B.

\section{Appendix B. KR adjoint-source (proofs)}

Throughout this Appendix, we use the notations of $\S 3.1$.

\section{Appendix B.1. Simplified case (thresholding constraint only)}

We first consider only the thresholding constraint in eqs. (11) and (14). It implies $\forall x \in X$ : $|\varphi(x)| \leq \lambda$, that can be split into two linear constraints $\varphi(x) \leq \lambda$ and $\varphi(x) \geq-\lambda$. Using the Lagrange multipliers method (Bertsekas, 1996), eq. (14) can be rewritten (dependencies to $\Delta f$ are implicit in the following to lighten the notations)

$$
\begin{aligned}
& \tilde{W}_{d}=\max _{\varphi} \min _{\alpha^{+} \geq 0, \alpha^{-} \geq 0} L\left(\varphi, \alpha^{+}, \alpha^{-}\right) \\
& L\left(\varphi, \alpha^{+}, \alpha^{-}\right)=\int_{X} \varphi(x) \Delta f(x) d \mu(x)-\int_{X}(\varphi(x)-\lambda) \alpha^{+}(x) d \mu(x)-\int_{X}(-\varphi(x)-\lambda) \alpha^{-}(x) d \mu(x) .
\end{aligned}
$$

Minimization of eq. (B.1) with respect to $\alpha^{+}$and $\alpha^{-}$gives the Karush-Kuhn-Tucker (KKT) conditions (Bertsekas, 1996)

$$
\begin{array}{ccc}
\varphi(x)=\lambda & \text { and } & \alpha^{+}(x)>0 \\
\text { or } \varphi(x)<\lambda & \text { and } & \alpha^{+}(x)=0 \\
-\varphi(x)=\lambda & \text { and } & \alpha^{-}(x)>0 \\
\text { or }-\varphi(x)<\lambda & \text { and } & \alpha^{-}(x)=0 .
\end{array}
$$


We deduce

$$
\begin{aligned}
& \alpha^{+}(x)>0 \Rightarrow \alpha^{-}(x)=0 \\
& \alpha^{-}(x)>0 \Rightarrow \alpha^{+}(x)=0 .
\end{aligned}
$$

Maximization of eq. (B.1) with respect to $\varphi$ gives almost everywhere in $X$

$$
\Delta f(x)=\alpha^{+}(x)-\alpha^{-}(x) .
$$

Combining eqs. (B.2)-(B.4), we deduce

$$
\begin{aligned}
& x \in X \backslash X_{\text {null }}^{(\Delta f)} \text { with } \Delta f(x)>0: \alpha^{+}(x)=\Delta f(x), \alpha^{-}(x)=0, \varphi_{\max }(x)=\lambda \\
& \Delta f(x)<0: \quad \alpha^{-}(x)=-\Delta f(x), \alpha^{+}(x)=0, \varphi_{\max }(x)=-\lambda .
\end{aligned}
$$

This result defines $\varphi_{\max }$ in $X \backslash X_{\text {null }}^{(\Delta f)}$, where it obviously remains stable under infinitesimal perturbations of $\Delta f$. Using the Lagrange multipliers to deduce this result in this simple case is somewhat "oversized". However, the method becomes interesting when considering more involved constraints as below, having the advantage to easilly generalize .

Appendix B.2. Intermediary case (1-Lipschitz constraint only, 1D coordinate space)

We here consider only the 1-Lipschitz constraint in eq. (14), and a 1D coordinate space $X$ that represents the time coordinate in practice. To lighten the notations, we consider a unit standarddeviation-like weight in eq. (11). From the considerations of Appendix A, the 1-Lipschitz constraint can be rewritten almost-everywhere in $X:\left|\frac{\partial \varphi(x)}{\partial x}\right| \leq 1$. This constraint can be split into two linear constraints $\frac{\partial \varphi(x)}{\partial x} \leq 1$ and $\frac{\partial \varphi(x)}{\partial x} \geq-1$. Using the Lagrange multipliers method (Bertsekas, 1996), eq. (14) can be rewritten

$$
\begin{aligned}
& \tilde{W}_{d}=\max _{\varphi} \min _{\beta^{+} \geq 0, \beta^{-} \geq 0} L\left(\varphi, \beta^{+}, \beta^{-}\right) \\
& \begin{aligned}
L\left(\varphi, \beta^{+}, \beta^{-}\right)= & \int_{X} \varphi(x) \Delta f(x) d \mu(x) \\
& \quad-\int_{X}\left(\frac{\partial \varphi(x)}{\partial x}-1\right) \beta^{+}(x) d \mu(x)-\int_{X}\left(-\frac{\partial \varphi(x)}{\partial x}-1\right) \beta^{-}(x) d \mu(x) .
\end{aligned}
\end{aligned}
$$

Minimization with respect to $\beta^{+}$and $\beta^{-}$gives the KKT conditions (Bertsekas, 1996)

$$
\begin{aligned}
& \frac{\partial \varphi(x)}{\partial x}=1 \quad \text { and } \quad \beta^{+}(x)>0 \\
& \text { or } \frac{\partial \varphi(x)}{\partial x}<1 \quad \text { and } \quad \beta^{+}(x)=0 \\
& -\frac{\partial \varphi(x)}{\partial x}=1 \quad \text { and } \quad \beta^{-}(x)>0 \\
& \text { or }-\frac{\partial \varphi(x)}{\partial x}<1 \quad \text { and } \quad \beta^{-}(x)=0 \text {. }
\end{aligned}
$$

We deduce

$$
\begin{aligned}
& \beta^{+}(x)>0 \Rightarrow \beta^{-}(x)=0 \text { and thus } \partial \beta^{-}(x) / \partial x=0 \\
& \beta^{-}(x)>0 \Rightarrow \beta^{+}(x)=0 \text { and thus } \partial \beta^{+}(x) / \partial x=0 .
\end{aligned}
$$

The "and thus" is due to the strong continuity of $\varphi$. For instance, if $\partial \varphi(x) / \partial x=1$, it cannot "jump" to $\partial \varphi\left(x^{\prime}\right) / \partial x^{\prime}=-1$ in the neighborhood of $x$. Thus, if $\beta^{+}(x)>0, \beta^{-}\left(x^{\prime}\right)$ must remain null in the neighborhood of $x$.

Considering $X=\left[x_{\min }, x_{\max }\right]$, maximization with respect to $\varphi$ gives almost everywhere in $X$

$$
\begin{aligned}
& \Delta f(x)+\left(\delta\left(x-x_{\min }\right)-\delta\left(x-x_{\max }\right)\right)\left(\beta^{+}(x)-\beta^{-}(x)\right)\left(\beta^{+}(x)-\beta^{-}(x)\right) \\
& =-\frac{\partial \beta^{+}(x)}{\partial x}+\frac{\partial \beta^{-}(x)}{\partial x} .
\end{aligned}
$$


Combining eqs. (B.8) and (B.9), we can deduce that always one of the constraints must saturate almost everywhere in $X \backslash X_{\text {null }}^{(\Delta f)}$ (the edges $x_{\min }$ and $x_{\max }$ of $X$ have null measure). More precisely, we have almost everywhere in $X \backslash X_{n u l l}^{(\Delta f)}$

$$
\begin{aligned}
x \in X \backslash X_{\text {null }}^{(\Delta f)}: & \frac{\partial \beta^{+}(x)}{\partial x}=-\Delta f(x) \Rightarrow \beta^{+}(x)>0, \beta^{-}(x)=0, \frac{\partial \varphi_{\max }(x)}{\partial x}=1 \\
& \text { or } \frac{\partial \beta^{-}(x)}{\partial x}=\Delta f(x) \Rightarrow \beta^{-}(x)>0, \beta^{+}(x)=0, \frac{\partial \varphi_{\max }(x)}{\partial x}=-1 .
\end{aligned}
$$

Complementarilly, integrating eq. (B.9) leads to

$$
\begin{aligned}
& -\Delta F_{\beta}(x)=\beta^{+}(x)-\beta^{-}(x) \\
& \Delta F_{\beta}(x)=\int_{x_{\text {min }}}^{x} \Delta f\left(x^{\prime}\right) d \mu\left(x^{\prime}\right)-\left(\beta^{+}\left(x_{\text {min }}\right)-\beta^{-}\left(x_{\text {min }}\right)\right) .
\end{aligned}
$$

Combining eqs. (B.8) and (B.11), we deduce almost everywhere in $X \backslash X_{\text {null }}^{\left(\Delta F_{\beta}\right)}$

$$
\begin{aligned}
x \in X \backslash X_{\text {null }}^{\left(\Delta F_{\beta}\right)} \text { with } \Delta F_{\beta}(x)<0: & \beta^{+}(x)=-\Delta F_{\beta}(x), \beta^{-}(x)=0, \frac{\partial \varphi_{\max }(x)}{\partial x}=1 \\
\Delta F_{\beta}(x)>0: & \beta^{-}(x)=\Delta F_{\beta}(x), \beta^{+}(x)=0, \frac{\partial \varphi_{\max }(x)}{\partial x}=-1 .
\end{aligned}
$$

Eq. (B.12) is satisfied in $X \backslash X_{\text {null }}^{\left(\Delta F_{\beta}\right)}$ and thus in $X \backslash X_{\text {null }}^{(\Delta f)}$ (as $X \backslash X_{\text {null }}^{\left(\Delta F_{\beta}\right)}$ tends to be "larger" for seismic data).

Obviously, eq. (B.9) (combined with eq. (B.8)), eq. (B.10) or eq. (B.12) remain stable under infinitesimal perturbations of $\Delta f$ in $X \backslash X_{n u l l}^{(\Delta f)}$. Also, eq. (B.9) (combined with eq. (B.8)) is sufficient to prove that always one of the constraints must saturate almost everywhere in $X \backslash X_{\text {null }}^{(\Delta f)}$. We introduced eqs. (B.10) and eq. (B.12) to give complementary insight.

Appendix B.3. Full case (1D coordinate space)

We now combine the two constraints (thresholding and 1-Lipschitz), still in the 1D coordinate space case and with unit standard-deviation-like weights. Using the Lagrange multipliers method (Bertsekas, 1996), we write

$$
\begin{aligned}
& \tilde{W}_{d}=\max _{\varphi} \min _{\alpha^{+} \geq 0, \alpha^{-} \geq 0, \beta^{+} \geq 0, \beta^{-} \geq 0} L\left(\varphi, \alpha^{+}, \alpha^{-}, \beta^{+}, \beta^{-}\right) \\
& \begin{aligned}
L\left(\varphi, \alpha^{+}, \alpha^{-}, \beta^{+}, \beta^{-}\right)= & \int_{X} \varphi(x) \Delta f(x) d \mu(x) \\
& -\int_{X}(\varphi(x)-\lambda) \alpha^{+}(x) d \mu(x)-\int_{X}(-\varphi(x)-\lambda) \alpha^{-}(x) d \mu(x) \\
& -\int_{X}\left(\frac{\partial \varphi(x)}{\partial x}-1\right) \beta^{+}(x) d \mu(x)-\int_{X}\left(-\frac{\partial \varphi(x)}{\partial x}-1\right) \beta^{-}(x) d \mu(x) .
\end{aligned}
\end{aligned}
$$

Minimization with respect to $\alpha^{+}, \alpha^{-}, \beta^{+}$and $\beta^{-}$gives KKT conditions (Bertsekas, 1996)

$$
\begin{array}{rrr}
\varphi(x)=\lambda & \text { and } & \alpha^{+}(x)>0 \\
\text { or } \varphi(x)<\lambda & \text { and } & \alpha^{+}(x)=0 \\
-\varphi(x)=\lambda & \text { and } & \alpha^{-}(x)>0 \\
\text { or }-\varphi(x)<\lambda & \text { and } & \alpha^{-}(x)=0 \\
\frac{\partial \varphi(x)}{\partial x}=1 & \text { and } & \beta^{+}(x)>0 \\
\text { or } \frac{\partial \varphi(x)}{\partial x}<1 & \text { and } & \beta^{+}(x)=0 \\
-\frac{\partial \varphi(x)}{\partial x}=1 & \text { and } & \beta^{-}(x)>0 \\
\text { or }-\frac{\partial \varphi(x)}{\partial x}<1 & \text { and } & \beta^{-}(x)=0 .
\end{array}
$$


None of these constraints can saturate simultaneously on non-null measure sets, as saturating the thresholding constraint implies a null derivative for $\varphi$ (and thus $\beta^{+}(x)=\beta^{-}(x)=0$ ) and saturating the 1-Lipschitz constraints implies a non-constant $\varphi$ (and thus $\left.\alpha^{+}(x)=\alpha^{-}(x)=0\right)$. We deduce almost everywhere

$$
\begin{aligned}
& \alpha^{+}(x)>0 \Rightarrow \alpha^{-}(x)=0, \beta^{+}(x)=\partial \beta^{+}(x) / \partial x=0, \beta^{-}(x)=\partial \beta^{-}(x) / \partial x=0 \\
& \alpha^{-}(x)>0 \Rightarrow \alpha^{+}(x)=0, \beta^{+}(x)=\partial \beta^{+}(x) / \partial x=0, \beta^{-}(x)=\partial \beta^{-}(x) / \partial x=0 \\
& \beta^{+}(x)>0 \Rightarrow \beta^{-}(x)=\partial \beta^{-}(x) / \partial x=0, \alpha^{+}(x)=0, \alpha^{-}(x)=0 \\
& \beta^{-}(x)>0 \Rightarrow \beta^{+}(x)=\partial \beta^{+}(x) / \partial x=0, \alpha^{+}(x)=0, \alpha^{-}(x)=0 .
\end{aligned}
$$

The justification of the configurations where the derivatives of $\beta^{+}$and $\beta^{-}$are null was already given after eq. (B.8).

Considering $X=\left[x_{\min }, x_{\max }\right]$, maximization with respect to $\varphi$ gives

$$
\begin{aligned}
& \Delta f(x)+\left(\delta\left(x-x_{\min }\right)-\delta\left(x-x_{\max }\right)\right)\left(\beta^{+}(x)-\beta^{-}(x)\right) \\
& =\alpha^{+}(x)-\alpha^{-}(x)-\frac{\partial \beta^{+}(x)}{\partial x}+\frac{\partial \beta^{-}(x)}{\partial x} .
\end{aligned}
$$

Combining eqs. (B.15) and (B.16), we can deduce that always one of the constraints must saturate almost everywhere in $X \backslash X_{\text {null }}^{(\Delta f)}$, and that eq. (B.16) remains stable under infinitesimal perturbations of $\Delta f$ in $X \backslash X_{n u l l}^{(\Delta f)}$.

Just to gain further insight, we introduce

$$
\begin{aligned}
& \Delta f_{\beta}(x)=\Delta f(x)+\frac{\partial \beta^{+}(x)}{\partial x}-\frac{\partial \beta^{-}(x)}{\partial x} \\
& \Delta F_{\beta, \alpha}(x)=\int_{x_{\text {min }}}^{x} \Delta f\left(x^{\prime}\right) d \mu\left(x^{\prime}\right)-\left(\beta^{+}\left(x_{m i n}\right)-\beta^{-}\left(x_{m i n}\right)\right)-\int_{x_{m i n}}^{x} d x^{\prime}\left(\alpha^{+}\left(x^{\prime}\right)-\alpha^{-}\left(x^{\prime}\right)\right) d \mu\left(x^{\prime}\right) .
\end{aligned}
$$

We then can deduce from eqs. (B.15)-(B.17)

$$
\begin{aligned}
\forall x \in X \backslash X_{\text {null }}^{\left(\Delta f_{\beta}\right)} \text { with } \Delta f_{\beta}(x)>0: & \alpha^{+}(x)=\Delta f_{\beta}(x), \alpha^{-}(x)=0, \varphi_{\max }(x)=\lambda \quad \text { (B.18) } \\
\Delta f_{\beta}(x)<0: & \alpha^{-}(x)=-\Delta f_{\beta}(x), \alpha^{+}(x)=0, \varphi_{\max }(x)=-\lambda \\
\forall x \in X \backslash X_{\text {null }}^{\left(\Delta F_{\beta, \alpha}\right)} \text { with } \Delta F_{\beta, \alpha}(x)<0: & \beta^{+}(x)=-\Delta F_{\beta, \alpha}(x), \beta^{-}(x)=0, \frac{\partial \varphi_{\max }(x)}{\partial x}=1 \\
\Delta F_{\beta, \alpha}(x)>0: & \beta^{-}(x)=\Delta F_{\beta, \alpha}(x), \beta^{+}(x)=0, \frac{\partial \varphi_{\max }(x)}{\partial x}=-1 .
\end{aligned}
$$

These equations define $\varphi_{\max }$ quite similarly than in eqs. (B.5) and (B.12), but they are here explicitly coupled. An implicit coupling is also imposed through the necessary (strong) continuity of $\varphi_{\max }$ and the fact that none of the constraints can saturate simultaneously, thus that

$$
X_{n u l l}=X_{\text {null }}^{\left(\Delta f_{\beta}\right)} \cup X_{\text {null }}^{\left(\Delta F_{\beta, \alpha}\right)} \quad \text { and } \quad \varnothing=X_{\text {null }}^{\left(\Delta f_{\beta}\right)} \cap X_{\text {null }}^{\left(\Delta F_{\beta, \alpha}\right)} .
$$

Obviously, eq. (B.16) (combined with eq. (B.15)) or eq. (B.18) remain stable under infinitesimal perturbations of $\Delta f$ in $X \backslash X_{\text {null }}^{(\Delta f)}$.

\section{Appendix B.4. Full case (2D coordinate space)}

We consider $X=\left[H_{i n l}^{\min }, H_{i n l}^{\max }\right] \times[0, T]$ with $x=\left(x_{i n l}, x_{t}\right)^{t}$, the results of Appendix A with nonunit standard-deviation-like weights and $d \rightarrow\|\|_{1}^{(X)}$. Eq. (B.16) becomes, with obvious notations

$$
\begin{aligned}
\Delta f(x) & +\sigma_{i n l}\left(\delta\left(x_{i n l}-H_{i n l}^{\text {min }}\right)-\delta\left(x_{i n l}-H_{i n l}^{\max }\right)\right)\left(\beta_{i n l}^{+}(x)-\beta_{i n l}^{-}(x)\right) \\
& +\sigma_{t}\left(\delta\left(x_{t}\right)-\delta\left(x_{t}-T\right)\right)\left(\beta_{t}^{+}(x)-\beta_{t}^{-}(x)\right) \\
& =\alpha^{+}(x)-\alpha^{-}(x)-\sigma_{i n l}\left(\frac{\partial \beta_{i n l}^{+}(x)}{\partial x_{i n l}}-\frac{\partial \beta_{i n l}^{-}(x)}{\partial x_{i n l}}\right)-\sigma_{t}\left(\frac{\partial \beta_{t}^{+}(x)}{\partial x_{t}}-\frac{\partial \beta_{t}^{-}(x)}{\partial x_{t}}\right) .
\end{aligned}
$$

Combining eq. (B.20) with the equivalent of eq. (B.15) in the $2 \mathrm{D}$ coordinate space case, we can deduce that always one of the constraints must saturate, almost everywhere in $X \backslash X_{\text {null }}^{(\Delta f)}$ and that eq. (B.20) remains stable under infinitesimal perturbations of $\Delta f$ in $X \backslash X_{\text {null }}^{(\Delta f)}$. The only subtlety 
is that, at a given position in $X \backslash X_{\text {null }}^{(\Delta f)}$ where the thresholding constraint does not saturate, only one of the Lipschitz constraints in the inline or time directions need to saturate, not necesarilly both. 


\section{References}

Ambrosio, L. and Gigli, N. (2009). A user's guide to optimal transport. Modelling and Optimisation of Flows on Networks, Part of the Lecture Notes in Mathematics book series, 2062:1-155.

Ambrosio, L., Gigli, N., and Savaré, G. (2005). Gradient Flows: In Metric Spaces and in the Space of Probability Measures. Birkhäuser Verlag, Basel.

Andersen, M. S., Dahl, J., and Vandenberghe, L. (2013). Cvxopt: A python package for convex optimization. abel. ee. ucla. edu/cvxopt.

Baek, H., Calandra, H., and Demanet, L. (2014). Velocity estimation via registration-guided leastsquares inversion. Geophysics, 79(2):R79-R89.

Bertsekas, D. (1996). Constrained Optimization and Lagrange Multiplier Methods. Academic Press, New York.

Brenders, A. J. and Pratt, R. G. (2007). Waveform tomography of marine seismic data: What can limited offset offer? 75th Annual International Meeting, SEG, Expanded Abstracts, pages 3024-3029.

Brezis, H. (2020). Analyse fonctionnelle: Théorie et applications. Dunod, Paris.

Bunks, C., Saleck, F. M., Zaleski, S., and Chavent, G. (1995). Multiscale seismic waveform inversion. Geophysics, 60(5):1457-1473.

Carotti, D., Hermant, O., Masclet, S., Reinier, M., Messud, J., Sedova, A., and Lambaré, G. (2020). Optimal transport full waveform inversion - Applications. 82nd EAGE Conference and Exhibition, Expanded Abstracts, Th Dome1 17.

Combettes, P. and Pesquet, J.-C. (2011). Proximal splitting methods in signal processing. Springer optimization and its applications: Springer New York, 49:185-212.

Engquist, B. and Froese, B. (2014). Application of the Wasserstein metric to seismic signals. Communications in Mathematical Sciences, 12(5).

Engquist, B., Froese, B., and Yang, Y. (2016). Optimal transport for seismic full waveform inversion. Communications in Mathematical Sciences, 14:2309-2330.

Hale, D. (2013). Dynamic warping of seismic images. Geophysics, 78(2):S105-S115.

Hanin, L. G. (1992). Kantorovich-rubinstein norm and its application in the theory of lipschitz spaces. Proceedings of the American Mathematical Society, 115(2):345-352.

Hermant, O., Aziz, A., Warzocha, S., and Al Jahdhami, M. (2020). Imaging complex fault structures on-shore oman using optimal transport full waveform inversion. 82nd EAGE Conference and Exhibition, Expanded Abstracts, We Dome1 19.

Hermant, O., Sedova, A., Royle, G., Retailleau, M., Messud, J., Lambaré, G., Al Abri, S., and Al Jahdhami, M. (2019). Broadband faz land data: an opportunity for FWI. 81st EAGE Conference and Exhibition, Workshop, WS08 11.

Kantorovich, L. V. (1942). On the translocation of masses. C. R. (Doklady) Acad. Sci. USSR, 321:199-201.

Kpadonou, F., Messud, J., Sedova, A., and Reinier, M. (2021). Optimal transport FWI with graph transform: Analysis and proposal of a partial shift strategy. Submitted to 83rd EAGE Conference and Exhibition.

Lailly, P. (1983). The seismic inverse problem as a sequence of before stack migrations. Conference on Inverse Scattering, Theory and Application, Society for Industrial and Applied Mathematics, Expanded Abstracts, 83:206-220.

Lellmann, J., Lorenz, D. A., Schonlieb, C., and T., V. (2014). Imaging with Kantorovich-Rubinstein discrepancy. SIAM J. Imaging Sci., 7(4):2833-2859.

Luo, S. and Sava, P. (2011). A deconvolution-based objective function for wave-equation inversion. 81th Annual International Meeting, SEG, Expanded Abstracts, pages 2788-2792.

Luo, Y. and Schuster, G. (1991). Wave-equation traveltime inversion. Geophysics, 56:645-653.

Martin, G., Wiley, R., and Marfurt, K. (2006). Marmousi2: an elastic upgrade for Marmousi. Leading Edge, 25:156-166. 
Messud, J. and Sedova, A. (2019). Multidimensional optimal transport for 3D FWI: Demonstration on field data. 81st EAGE Conference and Exhibition, Expanded Abstracts, Tu R08 02.

Métivier, L., Allain, A., Brossier, R., Mérigot, Q., Oudet, E., and Virieux, J. (2018). A graph-space approach to optimal transport for full waveform inversion. 88th Annual International Meeting, SEG, Expanded Abstracts, pages 1158-1162.

Métivier, L., Brossier, R., Mérigot, Q., and Oudet, E. (2019). A graph space optimal transport distance as a generalization of $L^{p}$-distances: application to a seismic imaging inverse problem. Inverse Problems, 35(8):085001.

Métivier, L., Brossier, R., Mérigot, Q., Oudet, E., and Virieux, J. (2016a). Increasing the robustness and applicability of full waveform inversion: an optimal transport distance strategy. The Leading Edge, 35:1060-1067.

Métivier, L., Brossier, R., Mérigot, Q., Oudet, E., and Virieux, J. (2016b). Measuring the misfit between seismograms using an optimal transport distance: Application to full waveform inversion. Geophysical Journal International, 205(1):345-377.

Métivier, L., Brossier, R., Mérigot, Q., Oudet, E., and Virieux, J. (2016c). An optimal transport approach for seismic tomography: application to 3D full waveform inversion. Inverse Problems, 32(11):115008.

Monge, G. (1781). Mémoire sur la théorie des déblais et des remblais. Histoire de l'Académie Royale des Sciences, pages 666-704.

Operto, S., Ravaut, C., Improta, L., Virieux, J., Herrero, A., and Dell'Aversana, P. (2004). Quantitative imaging of complex structures from multifold wide aperture seismic data: A case study. Geophysical Prospecting, 52:625-651.

Plessix, R.-E. (2006). A review of the adjoint-state method for computing the gradient of a functional with geophysical applications. Geophysical Journal International, 167(2):495-503.

Plessix, R.-E. (2009). 3D frequency-domain full-waveform inversion with an iterative solver. Geophysics, 74:WC149-WC157.

Poncet, R., Messud, J., Bader, M., Lambaré, G., Viguier, G., and Hidalgo, C. (2018). FWI with optimal transport: a 3D implementation and an application on a field dataset. 80th EAGE Conference and Exhibition, Expanded Abstracts, We A12 02.

Pratt, R. G. (1990). Inverse theory applied to multi-source cross-hole tomography. part ii: Elastic wave-equation method. Geophysical Prospecting, 38:311-330.

Pratt, R. G. (1999). Seismic waveform inversion in the frequency domain, part i: Theory and verification in a physical scale model. Geophysics, 64:888-901.

Rudin, W. (1991). Functional analysis (second edition). Dunod, Paris.

Sedova, A., Messud, J., Prigent, H., Masclet, S., Royle, G., and Lambaré, G. (2019). Acoustic land full waveform inversion on a broadband land dataset: the impact of optimal transport. $81 s t$ EAGE Conference and Exhibition, Expanded Abstracts, Th R08 07.

Shin, C. and Ha, W. (2008). A comparison between the behavior of objective functions for waveform inversion in the frequency and Laplace domains. Geophysics, 73(5):VE119-VE133.

Sirgue, L., Barkved, O., Van Gestel, J., Askim, O., and Kommedal, J. (2009). 3D waveform inversion on valhall wide-azimuth obc. 71st EAGE Conference and Exhibition, Extended abstracts, U038.

Sirgue, L. and Pratt, R. G. (2004). Efficient waveform inversion and imaging: A strategy for selecting temporal frequencies. Geophysics, 69:231-248.

Sun, B. and Al Khalifah, T. (2019). The application of an optimal transport to a preconditioned data matching function for robust waveform inversion. Geophysics, 84:R923-R945.

Tang, Y., Sun, B., and Al Khalifah, T. (2020). Wave-equation migration velocity analysis via the optimal-transport-based objective function. 90th Annual International Meeting, SEG, Expanded Abstracts, pages 3709-3713.

Tarantola, A. (1984). Inversion of seismic reflection data in the acoustic approximation. Geophysics, 49:1259-1266. 
Tarantola, A. (2005). Inverse Problem Theory and Methods for Model Parameters Estimation. Elsevier Science Publishers, Amsterdam.

Van Leeuwen, T. and Mulder, W. (2010). A correlation-based misfit criterion for wave-equation traveltime tomography. Geophysical Journal International, 182(3):1383-1394.

Vigh, D. and Starr, E. W. (2007). 3D pre-stack plane wave full waveform inversion. 75th Annual International Meeting, SEG, Expanded Abstracts, pages 1830-1834.

Villani, C. (2003). Topics in optimal transportation, volume 58 of Graduate Studies in Mathematics. American Mathematical Society.

Villani, C. (2008). Optimal transport: old and new, volume 338. Springer Science \& Business Media.

Virieux, J. and Operto, S. (2009). An overview of full-waveform inversion in exploration geophysics. Geophysics, 74(6):WCC127-WCC152.

Wang, D. and Wang, P. (2019). Adaptive quadratic Wasserstein full-waveform inversion. 89th Annual International Meeting, SEG, Expanded Abstracts, pages 1300-1304.

Wang, M., Xie, Y., Xu, W., Xin, K. F., Chuah, B. L., Loh, F. C., Manning, T., and Wolfarth, S. (2016). Dynamic-warping full-waveform inversion to overcome cycle skipping. SEG, pages $1273-1277$.

Warner, M. and Guasch, L. (2016). Adaptive waveform inversion: Theory. Geophysics, 81(6):R429R445.

Warner, M., Ratcliffe, A., Nangoo, T., Morgan, J., Umpleby, A., Shah, N., Vinje, V., Štekl, I., Guasch, L., Win, C., et al. (2013). Anisotropic 3D full-waveform inversion. Geophysics, 78(2):R59-R80.

Yang, Y. and Engquist, B. (2018). Analysis of optimal transport and related misfit functions in full-waveform inversion. Geophysics, 83(1):A7-A12.

Yang, Y., Engquist, B., Sun, J., and Froese, B. (2018). Application of optimal transport and the quadratic Wasserstein metric to full-waveform inversion. Geophysics, 83:R43-R62. 